\title{
REDESCRIPTIONS OF TWO SPECIES OF SEPIA (CEPHALOPODA: SEPIIDAE) FROM SOUTH AFRICAN WATERS: SEPIA ROBSONI (MASSY, 1927) AND S. FAUREI ROELEVELD, 1972
}

\author{
MAREK R. LIPIŃSKI
}

\begin{abstract}
Department of Ichthyology and Fisheries Science (DIFS), Rhodes University, P.O. Box 94, 6140 Grahamstown, South Africa

South African Institute of Aquatic Biodiversity (SAIAB), 11 Somerset Rd., 6140 Grahamstown, South Africa (e-mail: lipinski@mweb.co.za); (1) https://orcid.org/0000-0001-9362-4610

ABSTRACT: Two species of cuttlefish: Sepia robsoni (Massy) and Sepia faurei Roeleveld, are redescribed based on sexually mature males and females of both species. They were previously known only from their holotypes: male and female, respectively. They belong to a distinct group of small-sized sepiids, all nearendemics of southern African waters. The knowledge of the systematics and biology of this group is still limited despite the long time since the first description (1875). This is because, inter alia, of their small size: not larger than $4 \mathrm{~cm}$ mantle length at maturity. Twenty-one individuals of S. robsoni described here were scattered from Port Nolloth area to the Tsitsikamma coast (bottom depth $<37-449 \mathrm{~m}$ ). Eight known individuals of $S$. faurei came from the eastern Agulhas Bank (bottom depth 116-184 m). S. robsoni can be identified by extremely thinly calcified cuttlebone (transparent); smooth skin of dorsal mantle and head; tips of first pair of arms thick, finger-like, devoid of suckers. S. faurei can be distinguished by the following combination of characters: thick, heavily calcified cuttlebone but with flat and fused inner cone; skin of dorsal mantle and head very densely covered by characteristic warts; thin, whip-like tips of first pair of arms devoid of suckers. All described specimens of both species are deposited in the South African Institute of Aquatic Biodiversity (SAIAB) and in Iziko, South African Museum (SAMC).
\end{abstract}

KEY WORDS: small cuttlefish; redescriptions; South African waters; Sepia; Digitosepia

\section{INTRODUCTION}

Previous contributions (LIPIŃSKI \& LESLIE 2018, LIPIŃSKI 2020) concerning small-sized Sepiidae from southern Africa have outlined possible directions of research upon systematics of these peculiar cephalopods. In particular, a new subgenus (Digitosepia) was proposed with the following characteristics: sepiids with a substantially modified cuttlebone, combined with tips of I pair of arms devoid of suckers. This work adds thorough descriptions of two species of this subgenus, known before only from their holotypes: Sepia robsoni (Massy, 1927) and Sepia faurei Roeleveld, 1972. The original description of S. robsoni (holotype ML $17 \mathrm{~mm}$, male, maturity stage not mentioned) is brief, but includes most important characters of diagnostic significance. The description (and illustration) of the tips of both arms I, which were devoid of suckers, was most notable. MASSY (1927) considered this character as a possible result of an accident and subsequent regeneration. This was impossible to verify until now, as there were no further records of S. robsoni. The same concerns the cuttlebone, as MASSY (1927) regarded her specimen as "totally dissolved", therefore she did not illustrate "the membranous part remaining". As pointed out by LIPIŃSKI (2020), cuttlebones of small-sized sepiids may provide crucial characters of this group (shared by all eight species known to date). It is therefore important to provide even partial details of their cuttlebone morphology. MASSY (1927) provided drawings of the general habitus of the species (dorsal and ventral aspect), club, arm and club suckers, beaks, I pair of arms and radula. 
The original description of $S$. faurei (holotype ML $21 \mathrm{~mm}$, female, maturity stage not mentioned) is fairly thorough, but with obvious limitations. ROELEVELD (1972) illustrated the general habitus (dorsal and ventral aspect), tip of arm of I pair, club, and cuttlebone (incomplete). She also provided a comparison of characters between S. robsoni, S. dubia and S. faurei (in a separate table).

\section{MATERIAL AND METHODS}

Methods of this study were already outlined by LIPIŃSKI \& LESLIE (2018) and LIPIŃSKI (2020). Some details are repeated here for clarity. Specimens for this study were collected using bottom trawls during demersal research surveys off the west and south coasts of South Africa by the research vessels RS Africana, R/V Dr Fridtjof Nansen, and some commercial trawlers (Fig. 1). Details of bottom trawl gear, trawling, sorting and processing of the catch, and references thereof were given in AXELSEN \& JOHNSEN (2014), although it should be noted that they erroneously recorded the codend mesh size of the gear deployed by the RS Africana; the correct mesh sizes are $112 \mathrm{~mm}$ codend with $35 \mathrm{~mm}$ small mesh lining.

Measurements and counts (see Table 1 for definitions) follow ROELEVELD (1972), ROELEVELD \& LILTVED (1985), and LU \& REID (1997) and were taken from preserved specimens. The following changes to the terms and definitions given in LIPIŃSKI \& LESLIE (2018: table 1) are made here.

1. There is a vague difference between CIRC (definition: Table 1) and TrRC (Transverse Row Count; LU \& REID 1997: 279); therefore, TrRC is re-
The aim of this paper (as the two preceding papers) was two-fold. One was to give detailed morphological descriptions or redescriptions of small cuttlefish, as their systematic status required. The second was to provide as much illustration of intra-specific variation in each species as possible, to facilitate identification in the field. Therefore, many illustrations are provided.

named LORC and defined as the number of suckers counted at midline along the length $(\mathrm{Tcl})$ of the club.

2. CES was imprecisely defined and not easy to follow; it is defined here as the total number of suckers along the border of the whole club (combined total count from both sides of the club).

3. Maximum width of cuttlebone (WL; used in many studies) is added to the measurements of small sepiids, defined as the greatest width of cuttlebone (perpendicular to $\mathrm{L}$ ).

4. TIP1AL is defined as the length of the suckerless tip of I pair of arms.

Dorsal (ML) and ventral (MLv) mantle length were measured to the nearest $\mathrm{mm}$ below using slide callipers. Fin length (FL) was measured by placing a thread along the base of the fin from the anterior edge and marking the position of the posterior end of the fin, the length of the thread was then measured on a metal ruler. All other measurements were taken using dividers or a graticule in a stereo dissecting microscope at $10 \times$ magnification. Sucker diameters were measured at $40 \times$ magnification. Measurements

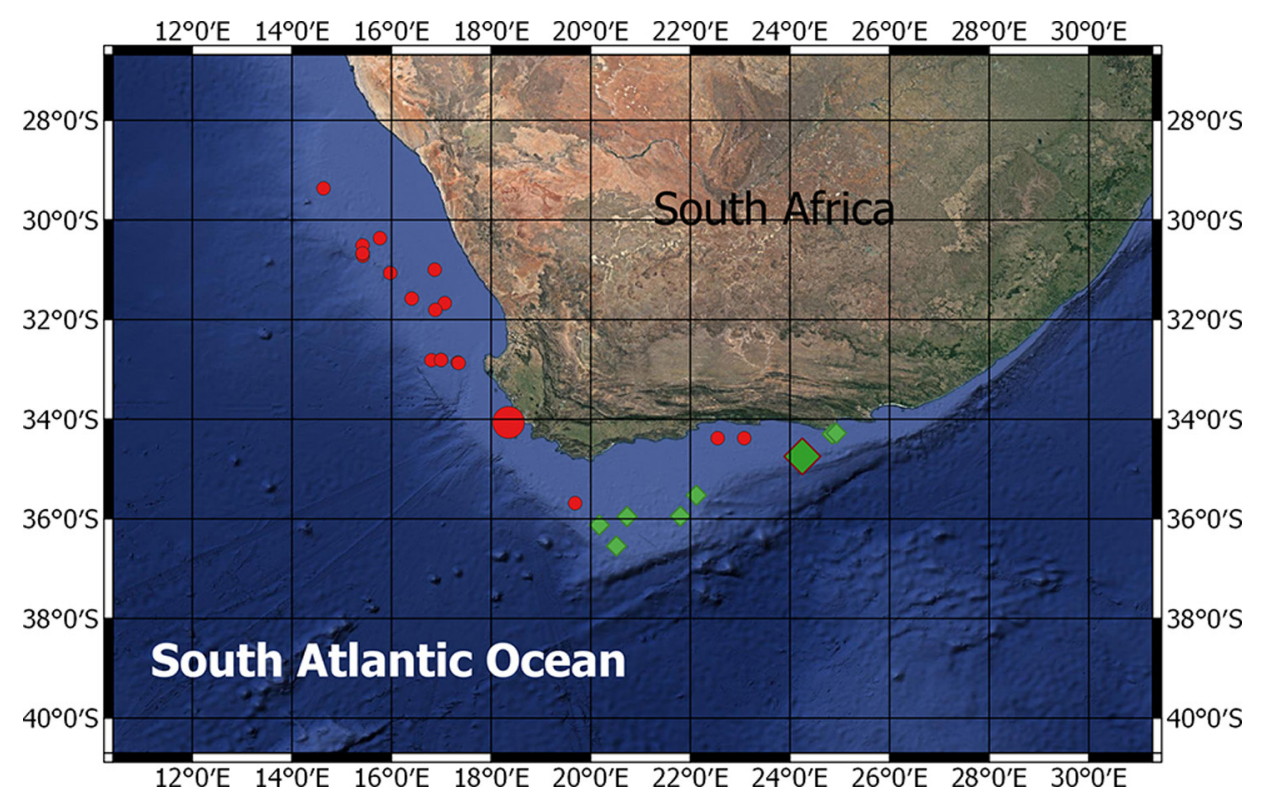

Fig. 1. Chart showing location of all survey trawls with localities of Sepia robsoni (circles; large circle: holotype), and $S$. faurei (diamonds; large diamond: holotype), during bottom trawl research surveys off the coast of South Africa 
and weights were taken from preserved specimens. Maturity was determined using the scale of LIPIŃSKI \& UNDERHILL (1995).

Most small sepiids in southern African waters have various complex skin structures which are important for correct identification of the different species. LIPIŃSKI \& LESLIE (2018) developed the follow- ing new definitions (or their new combinations) for some of these structures:

1. Warts are simple, solid, rounded, flat, and low excrescences on the skin; they may be quite large. Typical examples are found on the skin of Sepia roeleveldi Lipiński, 2020.

2. Tubercles are simple projections or protuberances, which are sharp or rounded. They may be very

Table 1. Description of measurements and counts. Definitions follow RoELEVELD (1972), ROELEVELD \& LILTVED (1985), LU \& REID (1997), LIPIŃSKI \& LESLIE (2018)

\begin{tabular}{ll}
\hline Abbreviation & \multicolumn{1}{c}{ Definition } \\
\hline AL1-AL4 & $\begin{array}{l}\text { Arm Length: length of the right (rt) or left (lt) arm of each designated (1 to 4) arm pair, measured } \\
\text { from the inner base of the most proximal sucker to the tip of the arm }\end{array}$ \\
AMH & $\begin{array}{l}\text { Anterior Mantle to Head: length of anterior projection of the dorsal mantle margin measured along } \\
\text { the midline from the anterior-most point of the dorsal mantle to the transverse line joining the } \\
\text { posterior-most points of the dorsal mantle margin on either side of the midline }\end{array}$
\end{tabular}

AS1-AS4 Arm Sucker diameter: diameter of the largest sucker on the right (rt) or left (lt) arm of each designated (1 to 4) arm pair

ASC1-ASC4 Arm Sucker Count: total number of suckers on the right (rt) or left (lt) arm of each designated (1 to 4) arm pair

ASl4 Arm Sucker left 4: diameter of the largest sucker on the hectocotylised (left ventral) arm

ASl4m Arm Sucker left 4 minimum: diameter of the smallest (modified) sucker on the hectocotylised arm

CES Club Edge Suckers: number of suckers along the whole edge of the club

ClRC Club Row Count: number of suckers in a single transverse row across the middle of the tentacular club

ClS Club Sucker diameter: diameter of the largest sucker on the tentacular club

CS\# Club Sucker count: total number of suckers on the tentacular club

CTR\# Club Transverse Row Number: number of transverse rows of suckers on the tentacular club

FFu Free Funnel length: measured from the anterior funnel opening to the dorsal attachment of the funnel to the head

FIa Fin Insertion anterior: distance from the anterior mantle margin to the anterior junction of fin and mantle

FIp Fin Insertion posterior: distance between the posterior junction of the left and right fins with the mantle

FL Fin Length: measured from anterior to posterior insertion along the curve of the mantle at the base of the fin

FuL Funnel Length: measured along the ventral midline from the anterior funnel opening to the posterior margin (the ventral mantle has to be cut to expose the posterior edge of the funnel)

FW Fin Width: measured from the lateral edge of the mantle to the free edge of the fin

HcL Hectocotylus Length: length of the hectocotylised (left ventral) arm measured from the inner base of the most proximal sucker to the tip of the arm

HL Head Length: measured from the anterior tip of the nuchal cartilage to the anterior edge of the dorsal interbranchial membrane between the dorsal arm pair

HW Head Width: the greatest width of the head (generally across the eyes)

$\mathrm{L} \quad$ Length of the cuttlebone along the mid-line

WL Width of the cuttlebone (where widest, perpendicular to L)

MHL Modified Hectocotylus Length: length of the modified (proximal) portion of the hectocotylus measured from the inner base of the most proximal sucker to the inner base of the first normal sucker

ML Mantle Length (dorsal): measured along the midline from the anterior edge of the dorsal mantle to the posterior end of the mantle

MLv Mantle Length (ventral): measured along the midline from the midpoint of the ventral emargination to the posterior end of the mantle

Tcl Tentacular club length: measured from the basal sucker to the tip of the club

TL Tentacle Length: measured from the point of emergence from the tentacular sac to the tip of the club

LORC Transverse Row Count: number of suckers in a single longitudinal row along the midline of the tentacular club

TIP1AL Tip of I pair Arms Length: measured from the last distal sucker to the tip of I pair of arms (left, right or both; second measurement in parentheses) 
long, or short, but never flat. Typical examples are found on the skin of S. faurei Roeleveld, 1972.

3. Papillae are prominent, complex protuberances in the following forms:

- Turrets (see RoELEVELd \& LiLTVEd 1985) where tubercles and/or warts are on top of each other; sometimes they form elaborate structures.

- Clusters where tubercles and/or warts are next to each other, forming a distinct unit.

- Patches where various structures (some or all defined above) are combined in one distinct unit (for example by elevated tissue). The term turret-cluster was proposed and defined earlier (LIPIŃSKI \& LESLIE 2018). However, the use of this term was found impractical in the reality of small sepiids. With the varied state of preservation of the material, and the large natural variation the term was difficult to recognise as such. Please note that the term "patch" was already used by ROELEVELD (1972: 254, 265).

Cuttlebone descriptions use standard terms (e.g. RoELEVELD 1972), except striae and septa. The use of "septa" is restricted here to the phragmocone and means not only walls between the chambers (there are hardly any chambers in small sepiids), but also layers of relatively thicker calcification within the phragmocone, which mark growth increments. "Striae" are used more loosely, mainly indicating growth lines of the dorsal shield. The relation of these marks to time is unknown and was not researched. The usage of the term "calcification" (used rather inconsistently in the sepiid literature) is explained in the Discussion.

Most of the photographs were taken using Canon EOS 7D Mk I camera and Canon EOS 650 camera coupled with Nikon stereomicroscope using a specially engineered ring.

Abbreviations for museums holding the material are: SAIAB - South African Institute of Aquatic Biodiversity, Grahamstown South Africa; SAMC Iziko, South African Museum in Cape Town, South Africa; BMNH - specimens at NHMUK catalogued prior to 1992; NHMUK - Natural History Museum London, UK.

\section{SYSTEMATIC ACCOUNTS}

\section{Sepia (Digitosepia) robsoni (Massy, 1927) (Figs 2-47, Table 2)}

Material. BMNH 1926.10.20.8. Holotype, mature male $17 \mathrm{~mm}$ ML, Hout Bay South Africa, 9-20 fms (i.e., $<36 \mathrm{~m}$ ), fine sand; gear: shrimp trawl. Condition poor. Examined: 1983 (LIPIŃSKI et al. 2000: 104). SAIAB 209568. Mature male $20 \mathrm{~mm}$ ML, TW 3.75 g. R/V Dr Fridtjof Nansen demersal survey 2005401 27 Feb. 2005, NA1061-112, 3249.02'S, 16 ${ }^{\circ} 48.00^{\prime} \mathrm{E}$, bottom trawl, $449 \mathrm{~m}$.

SAIAB 209569. Mature male $19 \mathrm{~mm}$ ML, TW $3.10 \mathrm{~g}$. Dr Fridtjof Nansen demersal survey 2012401, 10 Feb. 2012, Trawl 78, $31^{\circ} 40.3^{\prime} \mathrm{S}, 17^{\circ} 04.1^{\prime} \mathrm{E}$, bottom trawl, $258 \mathrm{~m}$.

SAIAB 209570. Male $16 \mathrm{~mm} \mathrm{ML,} \mathrm{TW} 2.12$ g. Dr Fridtjof Nansen demersal survey, 24 April 2004, TS823, $29^{\circ} 22.02^{\prime} \mathrm{S}, 14^{\circ} 37.98^{\prime} \mathrm{E}$, bottom trawl, $325 \mathrm{~m}$. SAIAB 209571. Male $15 \mathrm{~mm} \mathrm{ML}$, TW $1.33 \mathrm{~g}$. R/V Dr Fridtjof Nansen demersal survey, 05 June 2000, Station AN0212-064-2230, $34^{\circ} 23^{\prime} \mathrm{S}, 22^{\circ} 33^{\prime} \mathrm{E}$, bottom trawl, $88 \mathrm{~m}$.

SAIAB 209572. Male $22 \mathrm{~mm} \mathrm{ML}$, TW $2.87 \mathrm{~g}$. R/V Dr Fridtjof Nansen demersal survey 2003401, 20 Jan. 2003, Station AN562-046-3174, 31 $00^{\circ} \mathrm{S}, 16^{\circ} 52^{\prime} \mathrm{E}$, bottom trawl, $227 \mathrm{~m}$.

SAIAB 209573. Male $18 \mathrm{~mm}$ ML, TW 2.85 g. R/V Dr Fridtjof Nansen demersal survey 2012401, 15 Feb. 2012, Trawl $102,31^{\circ} 04^{\prime} \mathrm{S}, 15^{\circ} 58.4^{\prime} \mathrm{E}$, bottom trawl $354 \mathrm{~m}$
SAIAB 209574. Male $22 \mathrm{~mm}$ ML, TW $3.35 \mathrm{~g}$, and mature female $26 \mathrm{~mm}$ ML, TW $6.20 \mathrm{~g}$. R/V Dr Fridtjof Nansen demersal survey 2012401, 07 Feb. 2012, Trawl 57, 32 48.7'S, $16^{\circ} 59.6^{\prime} \mathrm{E}$, bottom trawl $350 \mathrm{~m}$. SAIAB 209575. Male $23 \mathrm{~mm}$ ML, TW $3.75 \mathrm{~g}$, and female $18 \mathrm{~mm} \mathrm{ML}$, TW $3.31 \mathrm{~g}$. R/V Dr Fridtjof Nansen demersal survey, 05 April 2007, Trawl 1400, $30^{\circ} 40.4^{\prime} \mathrm{S}, 15^{\circ} 25.0^{\prime} \mathrm{E}$, bottom trawl, $346 \mathrm{~m}$.

SAIAB 209576. Female 17 mm ML, TW 1.87 g. R/V Dr Fridtjof Nansen demersal survey 2008401, 24 Feb. 2008, Trawl $1575,32^{\circ} 51.8^{\prime} \mathrm{S}, 17^{\circ} 19.8^{\prime} \mathrm{E}$, bottom trawl $306 \mathrm{~m}$.

SAIAB 209577. Female 20 mm ML, TW 3.67 g. R/S Africana demersal survey, 21 April 1999, A19566050-3470, 34 $23^{\circ} \mathrm{S}, 23^{\circ} 05^{\prime} \mathrm{E}$, bottom trawl, $101 \mathrm{~m}$.

SAIAB 209578. Female $27 \mathrm{~mm}$ ML, TW $6.18 \mathrm{~g}$. R/V Dr Fridtjof Nansen demersal survey, 07 April 2007, Trawl 1405, 313․ 34 'S, $16^{\circ} 24.3^{\prime} \mathrm{E}$, bottom trawl, $366 \mathrm{~m}$. SAIAB 209579. Female $22 \mathrm{~mm}$ ML, TW $4.04 \mathrm{~g}$. R/V Dr Fridtjof Nansen demersal survey, 09 Jan. 2010, Trawl 9, 35 $41.3^{\prime} \mathrm{S}, 19^{\circ} 41.2^{\prime} \mathrm{E}$, bottom trawl, $183 \mathrm{~m}$. SAIAB 209580. Female 23 mm ML, TW 6.31 g. M/T Andromeda demersal survey, 01 March 2015, Station D00429 (trawl 064, grid 4078-58125), 31 $1^{\circ} 48.4^{\prime} \mathrm{S}$, $16^{\circ} 52.7^{\prime} \mathrm{E}$, bottom trawl, $290 \mathrm{~m}$.

SAIAB 209581. Female 20 mm ML, TW 2.66 g. R/V Dr Fridtjof Nansen demersal survey, 07 Feb. 2013, Trawl 116, 30³0.8'S, $15^{\circ} 25.0^{\prime} \mathrm{E}$, bottom trawl, $301 \mathrm{~m}$. SAIAB 209582. Female $26 \mathrm{~mm}$ ML, TW $5.12 \mathrm{~g}$. R/V Dr Fridtjof Nansen demersal survey, 05 April 2007, 


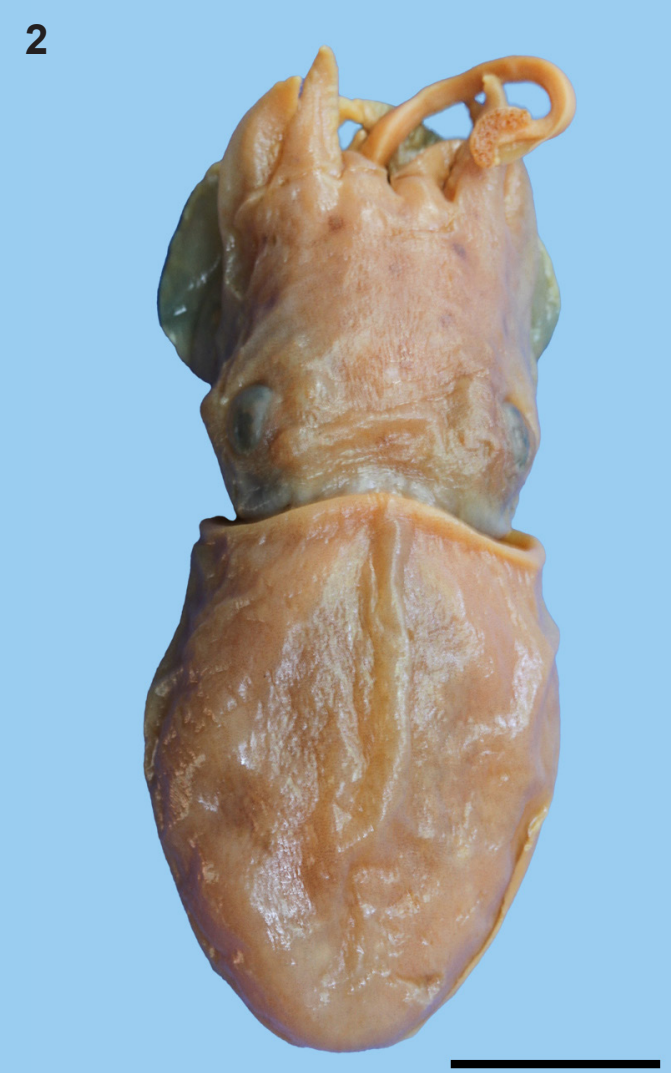

\section{3}

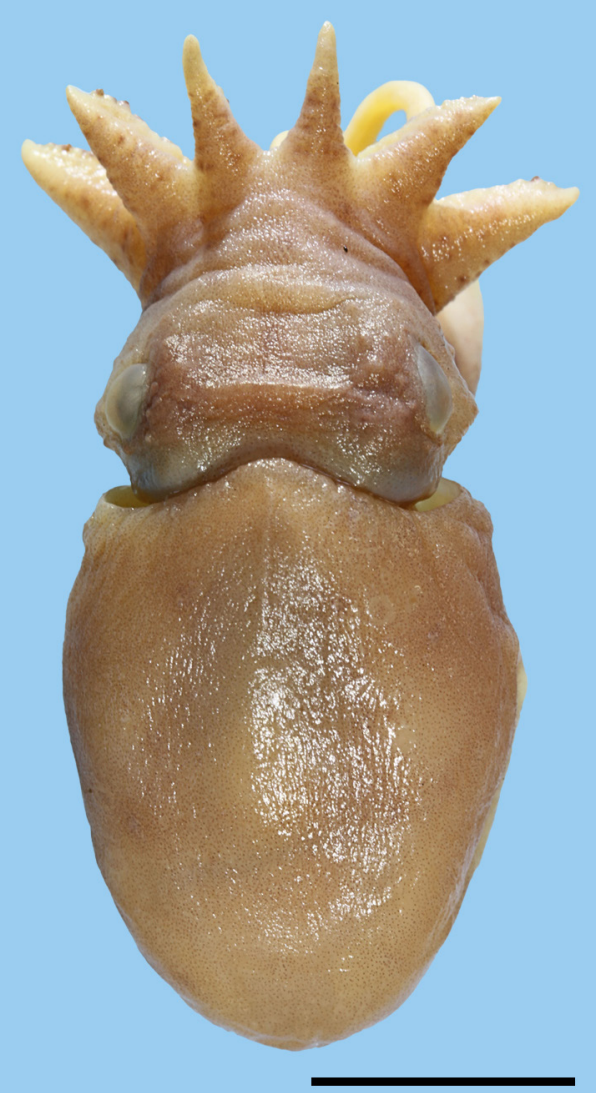

4

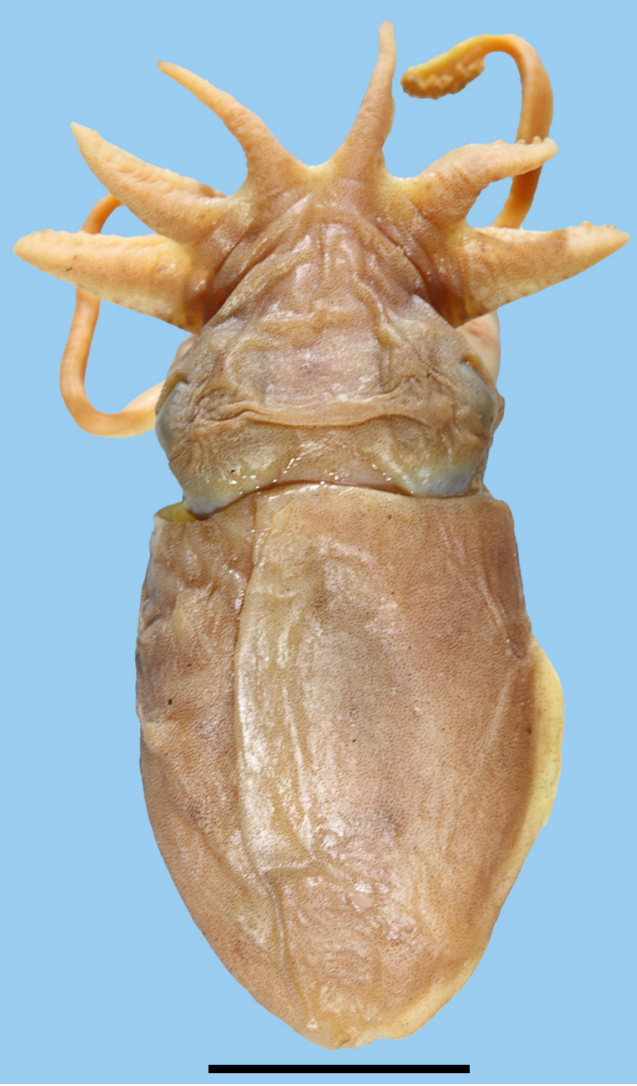

5

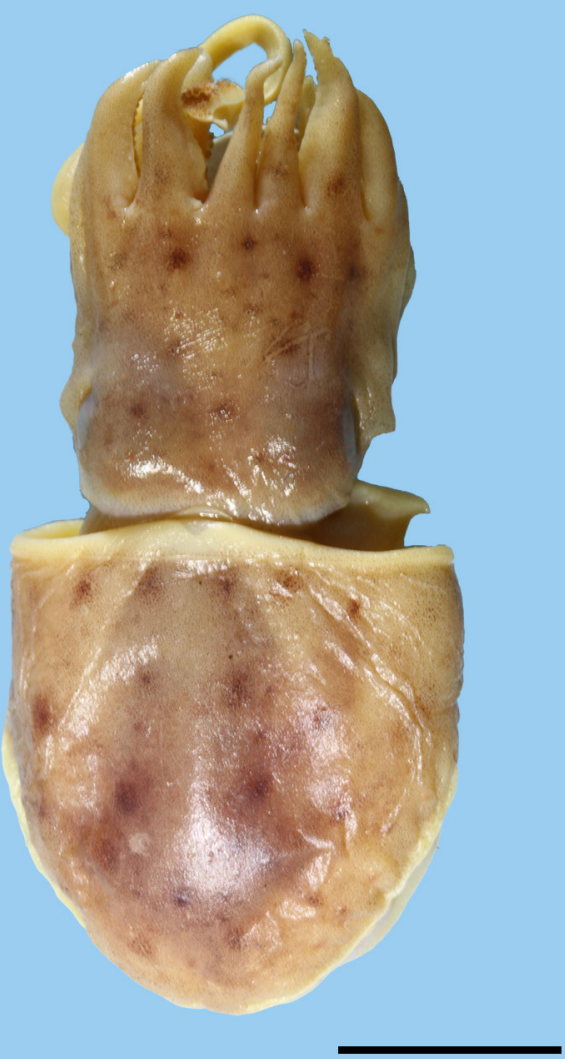

Figs 2-5. Sepia robsoni, dorsal view: 2 - SAIAB 209578, female 27 mm ML; 3 - SAIAB 209574a, male 22 mm ML; 4 - SAIAB 209572, male 22 mm ML; 5 - SAIAB 209579, female $22 \mathrm{~mm}$ ML. Scale bars $10 \mathrm{~mm}$ 

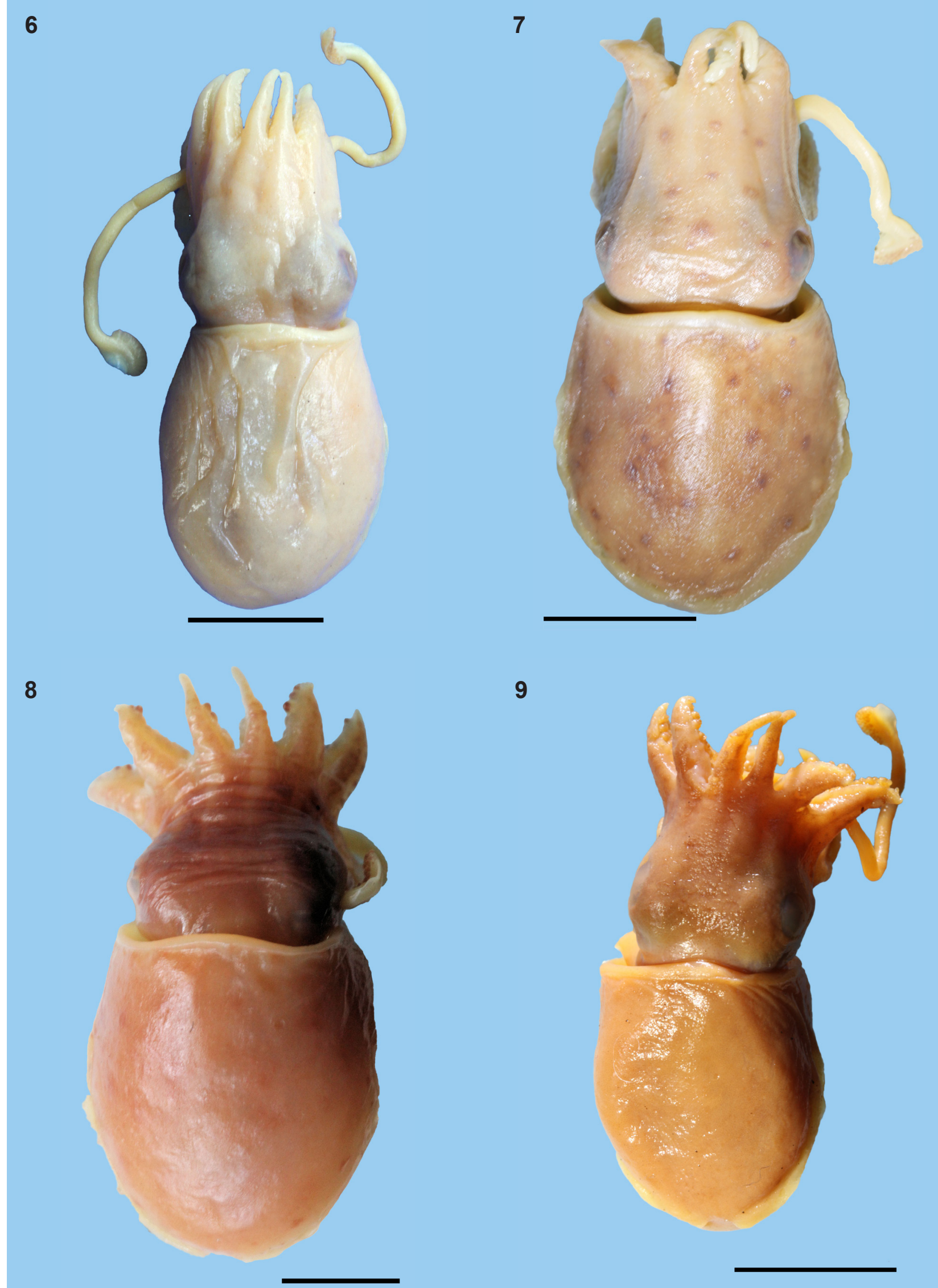

9

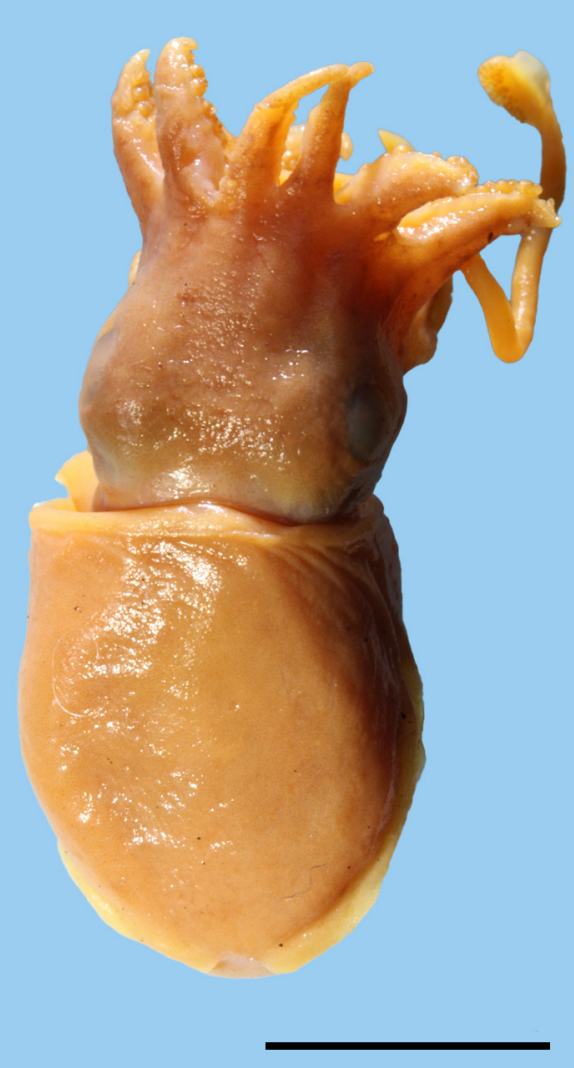

Figs 6-9. Sepia robsoni, dorsal view: 6 - SAIAB 209584, female 22 mm ML; 7 - SAIAB 209577, female 20 mm ML; 8 SAIAB 209571, male 14 mm ML; 9 - SAIAB 209570, male 16 mm ML. Scale bars: 10 mm (6-7, 9), 5 mm (8) 
Trawl 1401, $30^{\circ} 40.7^{\prime} \mathrm{S}, \quad 5^{\circ} 25.2^{\prime} \mathrm{E}$, bottom trawl, SAIAB 209584. Mature female $22 \mathrm{~mm} \mathrm{ML}$, TW 4.34 $346 \mathrm{~m}$.

SAIAB 209583. Female $21 \mathrm{~mm}$ ML, TW $3.58 \mathrm{~g} . \mathrm{R} / \mathrm{V}$ Dr Fridtjof Nansen demersal survey, 05 April 2007, Trawl 1399, $30^{\circ} 43.3^{\prime} \mathrm{S}, 1^{\circ} 25.4^{\prime} \mathrm{E}$, bottom trawl, $399 \mathrm{~m}$.

g. R/V Dr Fridtjof Nansen demersal survey, $21 \mathrm{Feb}$. 2001, AN0320-75-3072, $30^{\circ} 22$ 'S, $15^{\circ} 46^{\prime} \mathrm{E}$, bottom trawl, $245 \mathrm{~m}$.

SAIAB 209585. Female 16 mm ML, TW 1.67 g. R/V Dr Fridtjof Nansen demersal survey, 30 Jan. 2011, Trawl 53, 32 $52.5^{\prime} \mathrm{S}, 17^{\circ} 20.8^{\prime} \mathrm{E}$, bottom trawl, $305 \mathrm{~m}$.

Table 2. Measurements (mm), weight $(\mathrm{g})$ and counts recorded for characters of the holotype and selected specimens of Sepia robsoni

\begin{tabular}{|c|c|c|c|c|c|c|c|}
\hline $\begin{array}{l}\text { Catalogue } \\
\text { number }\end{array}$ & $\begin{array}{c}\text { NHMUK } \\
\text { 1926.10.20.8 } \\
\text { Holotype }\end{array}$ & $\begin{array}{l}\text { SAIAB } \\
209568\end{array}$ & $\begin{array}{l}\text { SAIAB } \\
209571\end{array}$ & $\begin{array}{l}\text { SAIAB } \\
209575\end{array}$ & $\begin{array}{l}\text { SAIAB } \\
209578\end{array}$ & $\begin{array}{l}\text { SAIAB } \\
209584\end{array}$ & $\begin{array}{l}\text { SAIAB } \\
209585\end{array}$ \\
\hline Sex & $\mathrm{M}$ & $\mathrm{M}$ & $\mathrm{M}$ & $\mathrm{M}$ & F & F & F \\
\hline Maturity & $\mathrm{V}$ & $\mathrm{V}$ & $*$ & $*$ & V & $\mathrm{V}$ & $*$ \\
\hline Weight & 1.22 & 3.75 & 1.33 & 3.75 & 6.18 & 4.34 & 1.67 \\
\hline ML & 16 & 20 & 15 & 23 & 27 & 22 & 16 \\
\hline MLv & 14 & 18 & 14 & 21 & 25 & 21 & $*$ \\
\hline $\mathrm{L}$ & $*$ & $*$ & $*$ & $*$ & $*$ & $*$ & * \\
\hline WL & $*$ & $*$ & $*$ & $*$ & $*$ & $*$ & $*$ \\
\hline $\mathrm{HL}$ & 6 & 14 & 9 & 13 & 16 & 15 & 10.5 \\
\hline HW & 9 & 13 & 9.5 & 13 & 16 & 13 & 11 \\
\hline AMH & 4 & 1.5 & 0.5 & 0 & 3 & 0 & $*$ \\
\hline FL & 12.5 & 14 & 11 & 20 & 20 & 17 & 9 \\
\hline FW & ${ }^{*}$ & 1 & 1.2 & 2 & 2 & 1.5 & 0.5 \\
\hline FIa & * & 7 & 5 & 7 & 10.5 & 7 & ${ }^{*}$ \\
\hline FIp & $*$ & 2 & 1.3 & 1.6 & 2.8 & 2.5 & 2.3 \\
\hline $\mathrm{FFu}$ & 2.3 & 5 & 5 & 6.5 & 6.5 & 4 & 4 \\
\hline FuL & 7 & 10 & $*$ & $*$ & 14 & 10 & 8 \\
\hline AL1-rt & 10 & 12 & 7 & 10.5 & 10 & 9 & 7 \\
\hline AL2-rt & 11 & 10.5 & 6 & 9.5 & 11 & 10 & 7.5 \\
\hline AL3-rt & 11 & 11.5 & 6.5 & 11 & 11 & 10 & 8.5 \\
\hline AL4-rt & 13 & 11 & 8 & 12.5 & 12.5 & 11 & 8.5 \\
\hline $\mathrm{HcL}$ & 8 & 12 & 8.5 & 11.5 & - & - & - \\
\hline MHL & ${ }^{*}$ & 8 & 6 & 7.5 & - & - & - \\
\hline TIP1AL & 1.2 & 4.5 & 2 & 3 & 4 & 3.5 & 2 \\
\hline $\mathrm{TL}$ & $*$ & 17 & 15 & 27 & 27 & 23 & 24 \\
\hline $\mathrm{Tcl}$ & 3 & 4 & 2.7 & 4.2 & 4 & 4.2 & 4 \\
\hline AS1-rt & 0.5 & 0.65 & 0.5 & 0.8 & 0.65 & 0.55 & 0.5 \\
\hline AS2-rt & 0.5 & 0.9 & 0.65 & 0.8 & 0.65 & 0.6 & 0.5 \\
\hline AS3-rt & 0.5 & 0.9 & 0.65 & 0.8 & 0.65 & 0.6 & 0.5 \\
\hline AS4-rt & $*$ & 0.9 & 0.6 & 1 & 0.60 & 0.45 & 0.45 \\
\hline ASl4 & * & 0.5 & 0.3 & 0.5 & - & - & - \\
\hline ASl4m & * & 0.25 & 0.18 & 0.2 & - & - & - \\
\hline $\mathrm{ClS}$ & 0.1 & 0.3 & 0.2 & 0.3 & 0.3 & 0.2 & 0.2 \\
\hline ASC1-rt & 14 & 19 & 16 & 16 & 17 & 20 & 19 \\
\hline ASC2-rt & 15 & 28 & 21 & 23 & 39 & 43 & 41 \\
\hline ASC3-rt & 21 & 28 & 21 & 22 & 40 & 46 & 38 \\
\hline ASC4-rt & $*$ & 30 & 29 & 28 & 32 & 41 & 31 \\
\hline CS\# & 52 & 50 & 46 & 44 & 48 & 46 & 49 \\
\hline CTR\# & $*$ & 16 & 13 & 13 & 12 & 13 & 13 \\
\hline CES & 29 & 26 & 28 & 27 & 29 & 26 & 28 \\
\hline CIRC & $3-5$ & $3-4$ & $3-4$ & $3-4$ & $3-4$ & $3-4$ & $3-4$ \\
\hline LORC & 13 & 13 & 14 & 14 & 13 & 13 & 12 \\
\hline
\end{tabular}

- - not applicable.

* - missing or impossible to measure.

Holotype was remeasured. 


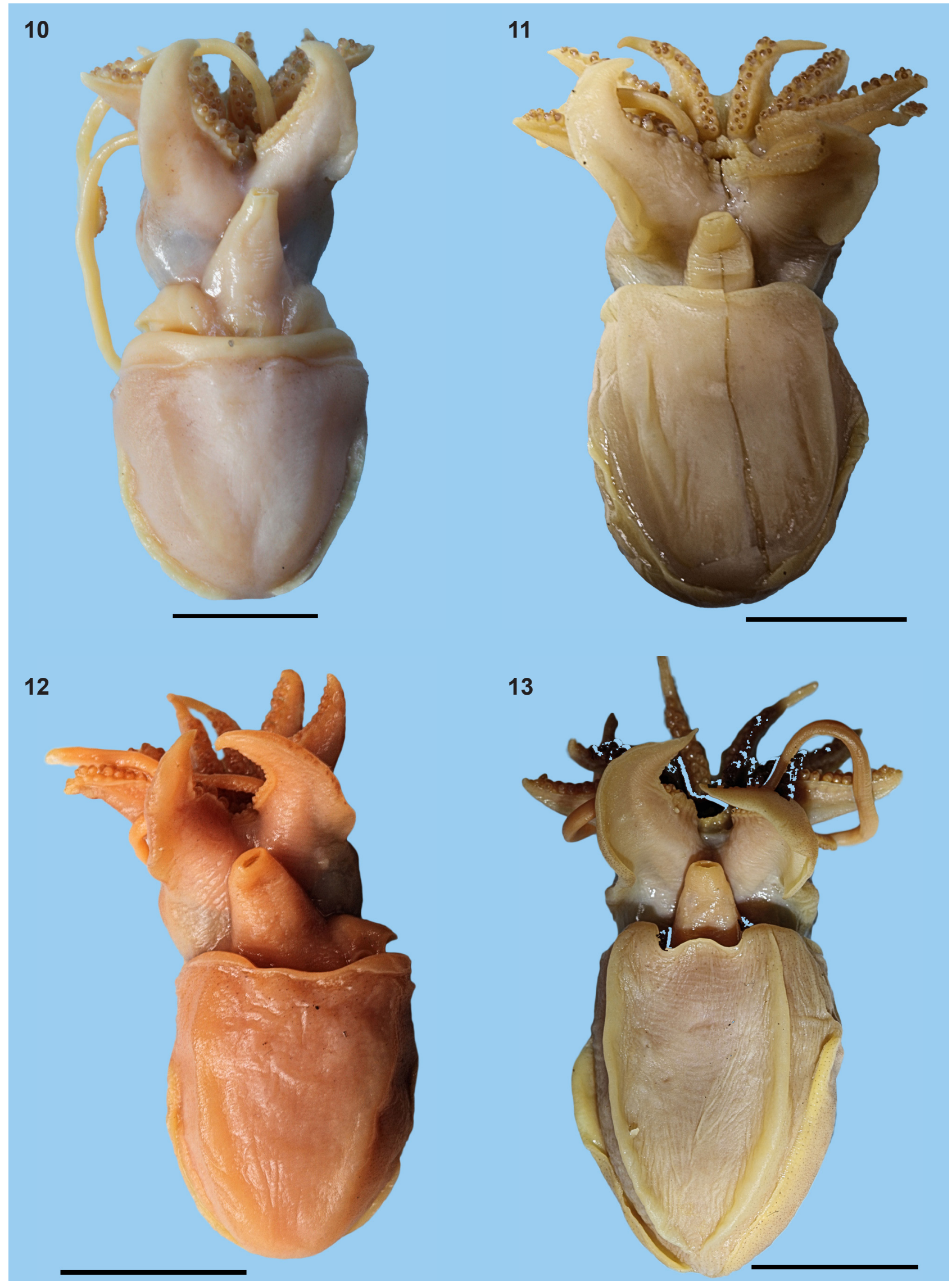

Figs 10-13. Sepia robsoni, ventral view: 10 - SAIAB 209573, male 18 mm ML; 11 - SAIAB 209568, male 20 mm ML; 12 SAIAB 209570, male 16 mm ML; 13 - SAIAB 209572, male 22 mm ML. Scale bars $10 \mathrm{~mm}$ 


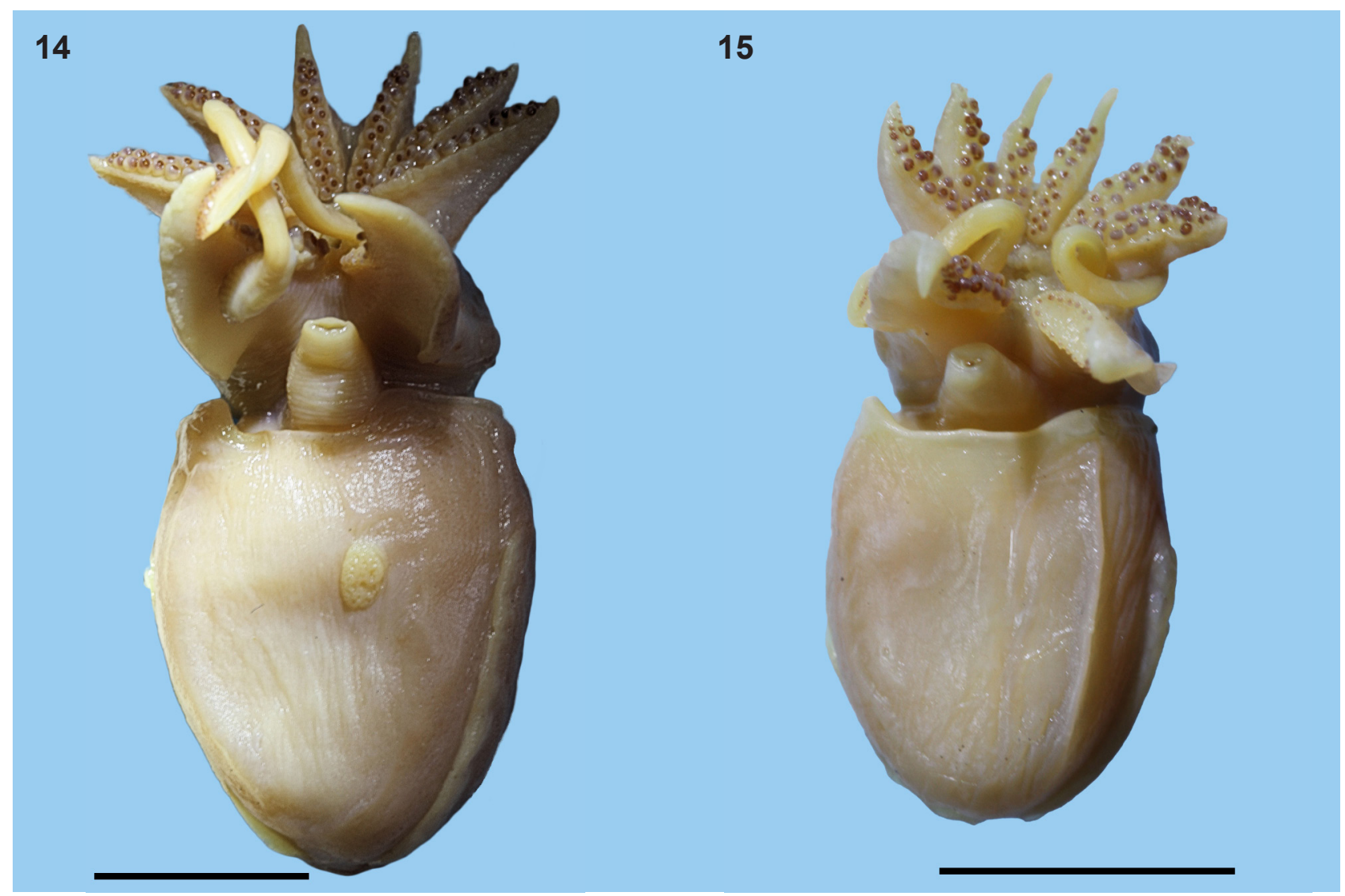

Figs 14-15. Sepia robsoni, ventral view: 14 - SAIAB 209574a, male 22 mm ML; 15 - SAIAB 209571, male 14 mm ML. Scale bars $10 \mathrm{~mm}$

Diagnosis. Cuttlebone extremely thinly calcified, mostly transparent (may be opaque in middle of phragmocone). Phragmocone reduced to thin plate fused to dorsal shield. Inner and outer cones flat, completely fused with surrounding structures. No forked limbs. No spine, but small distinct terminal knob (different colour, but transparent). Skin of dorsal mantle and head smooth (there may be 2-3 small warts: irregularly distributed, usually on mantle margins). Tips of I pair of arms thick, finger-like, very prominent, devoid of suckers.

Description. Small at maturity; eight known males (all adult) $14-23 \mathrm{~mm}$, and 12 females (maturity III-V) 16-27 mm (Table 2). Mantle squat, globose, oval (rounder in smaller animals), dorso-anterior margin more often than not wide $\Lambda$-shape (Figs 2-5), but may be also straight (Figs 6-9). Ventro-anterior margin either straight (Fig. 11) or emarginated (inverted trapezoid; may be very wide) in both sexes (Figs 1215). Ventral mantle margins with distinct keels (Fig. 13). Fins narrow, ending well before anterior mantle margin (FIa 30-39\% ML; Figs 16-18); small gap between fins posteriorly. Colour of dorsal mantle and head in preserved (10\% formalin followed by $70 \%$ ethyl alcohol) specimens variable, light grey with darker speckles or reddish brown (Figs 2-9). Skin on dorsal mantle, head and arms uniformly smooth, shiny (Figs 2-9); few small warts or simple tubercles may be present, usually marginally.

Head width variable: equal to mantle opening width or narrower, square, rarely elongated; eyes large, dorso-lateral or sometimes lateral; neck wide (Figs 2-9). Tentacle pouch large and deep. Buccal membrane without suckers.

Arms robust, stout, of varied length (Figs 19-22, Table 2), relatively short, strong membrane joining pairs I-IV proximally for about $30-50 \%$ of arm length, absent ventrally between arms IV (Figs 19, 22) in both sexes. Protective membranes well developed, fleshy, but also variable, tend to be more prominent in large females (Figs 19-22). Suckers globular, bi-serial on all arms; sucker rows closely spaced in males, they may be wider apart in females (Figs 19-22). Diameters of suckers variable (Table 2); they change rather abruptly along arms III-IV in females (after 6-10th pair of suckers and again after 2-4th pair of suckers). Sucker rings on arms smooth, and on club with tiny teeth (Fig. 24). Tips of arms of I pair devoid of suckers, thick, fleshy, finger-like, with narrow cleft in oral mid-line; sometimes knobby in appearance. Tips may be of different length on each arm (range $29-40 \%$ of length on I pair arm) (Figs 25-27). Tips of arms IV in the large female (SAIAB 209578) also devoid of suckers (Fig. 23); frequency 
16

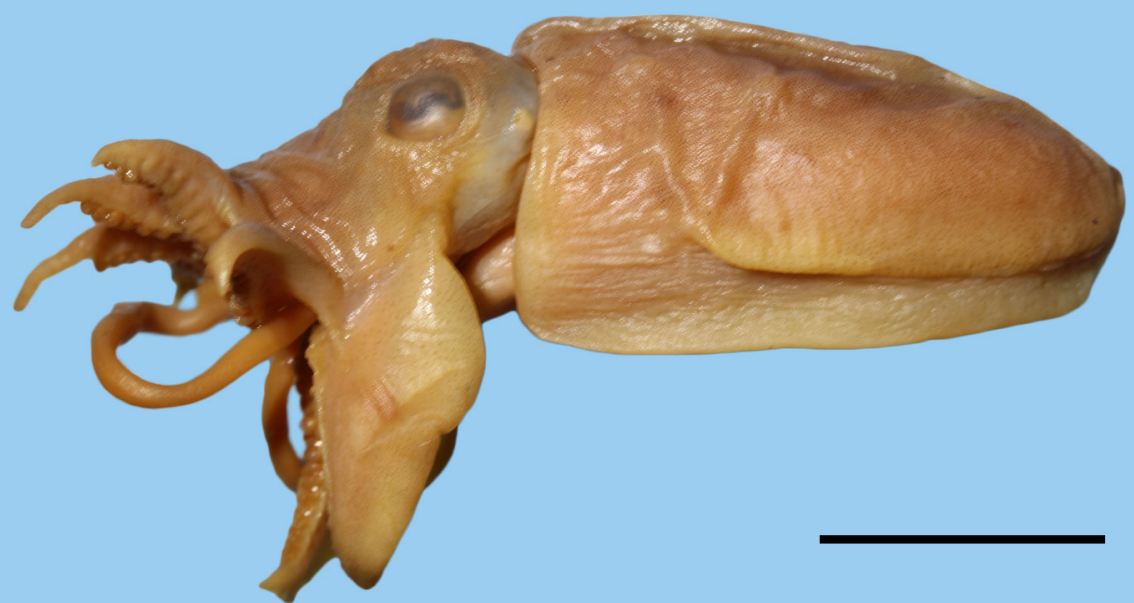

17

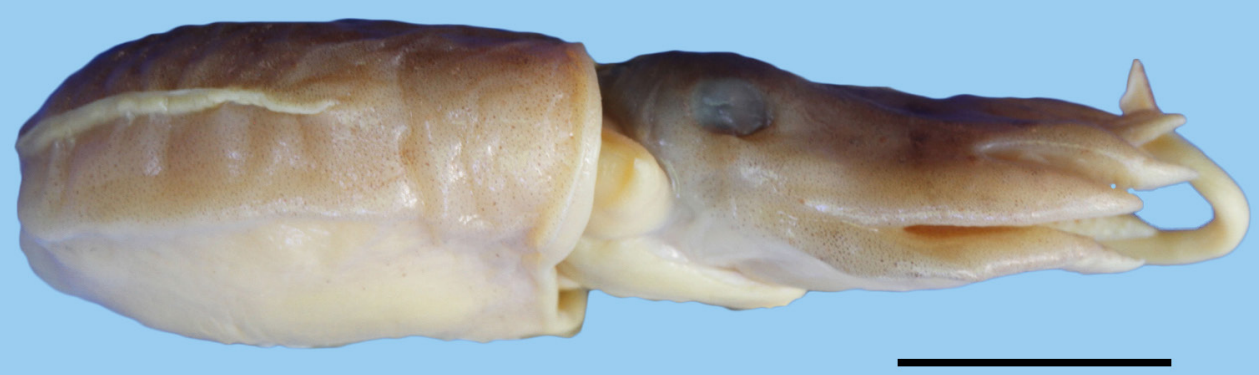

18

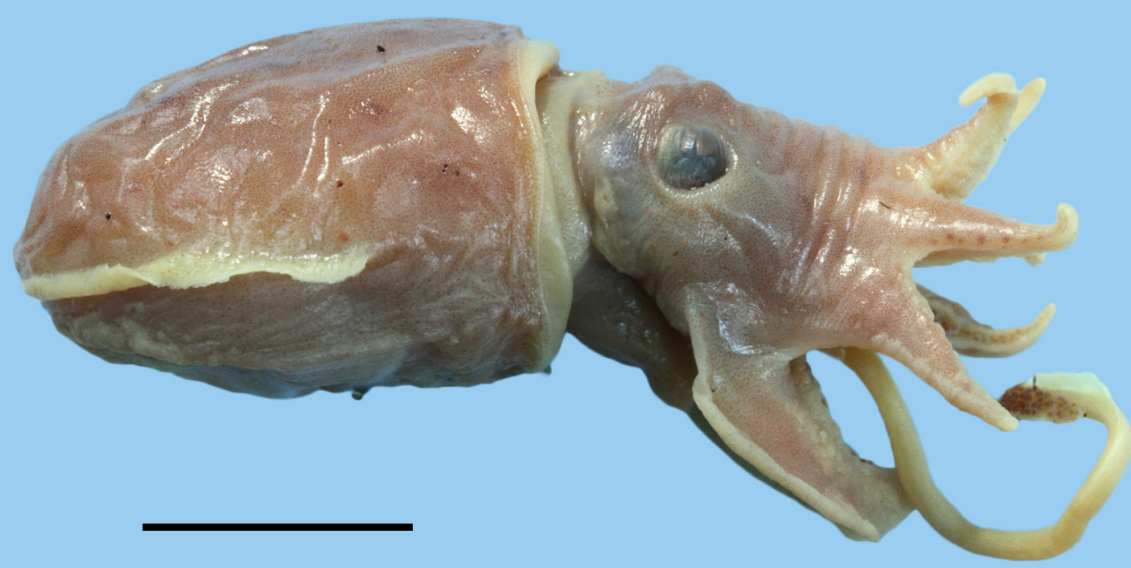

Figs 16-18. Sepia robsoni, lateral view: 16 - SAIAB 209572, male 22 mm ML; 17 - SAIAB 209579, female 22 mm ML; 18 SAIAB 209583, female $21 \mathrm{~mm}$ ML. Scale bars $10 \mathrm{~mm}$ 


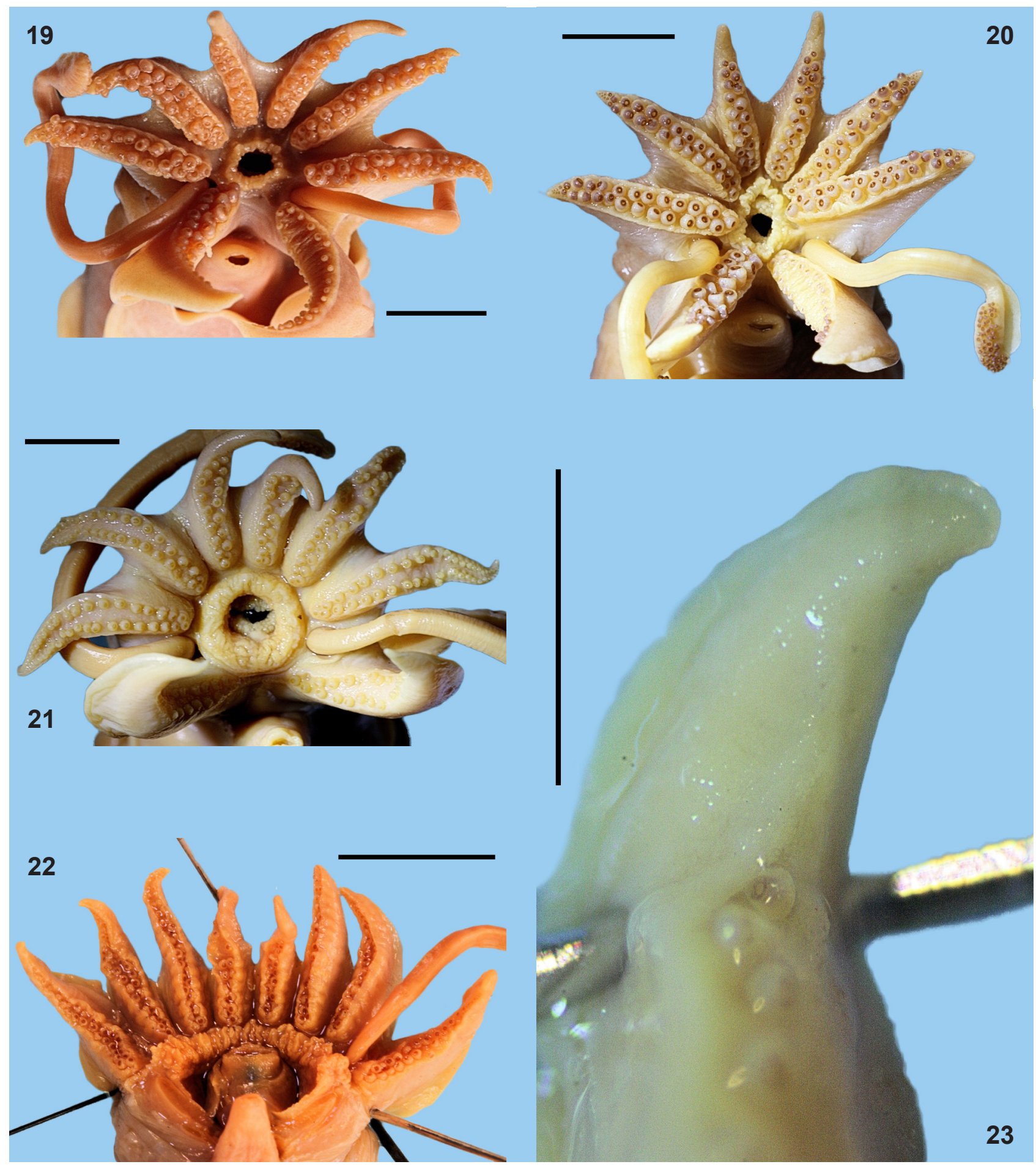

Figs 19-23. Sepia robsoni, arms: 19 - SAIAB 209572, male 22 mm ML; 20 - SAIAB 209574a, male 22 mm ML; 21 - SAIAB 209580, female 23 mm ML; 22-23 - SAIAB 209578, female $27 \mathrm{~mm} \mathrm{ML:} 23$ - tip of IV right arm. Scale bars $5 \mathrm{~mm}$ (19-21), $10 \mathrm{~mm}(22), 1 \mathrm{~mm}(23)$

of occurrence of this character has to be assessed on a larger material than currently available. Arms and especially their armature modified in mature males, suckers enlarged on arms I-IV except 2-4 terminal sucker pairs (Figs 19-21). Left ventral arm IV hectocotylised (Fig. 28): single basal sucker large, subsequent pairs become gradually smaller (on ventral margin much smaller); there are 11 pairs of marginal suckers, each marginal line straight; then five pairs of enlarged suckers (last much smaller than the others of the five pairs; Fig. 29) and one small distal sucker. Hectocotylus tip with tiny bi-serial small suckers (Fig. 29). Fleshy transverse folds running alternately between small marginal sucker pairs (dorsal sucker 

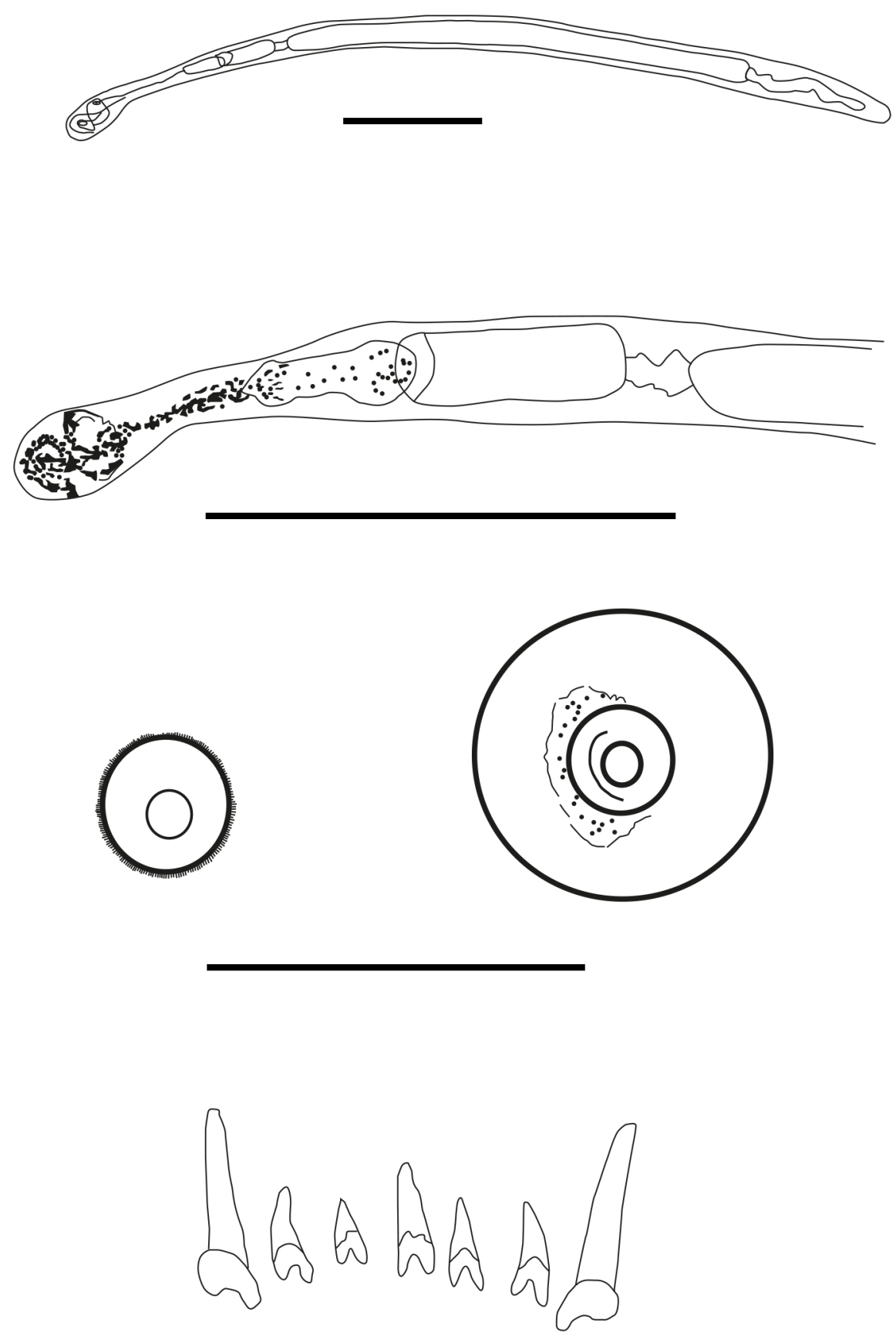

Fig. 24. Sepia robsoni, spermatophore and suckers: top - spermatophore, SAIAB 209568, male 20 mm ML; second from top - anterior part of spermatophore magnified; third from top (left) - club sucker (middle of central transverse row) and arm IV sucker, 3rd pair from base (suckers viewed from above), all suckers from SAIAB 209582, female $26 \mathrm{~mm}$ ML; bottom - radula, SAIAB 209582, female $26 \mathrm{~mm}$ ML. Scale bars: $0.5 \mathrm{~mm}$ 


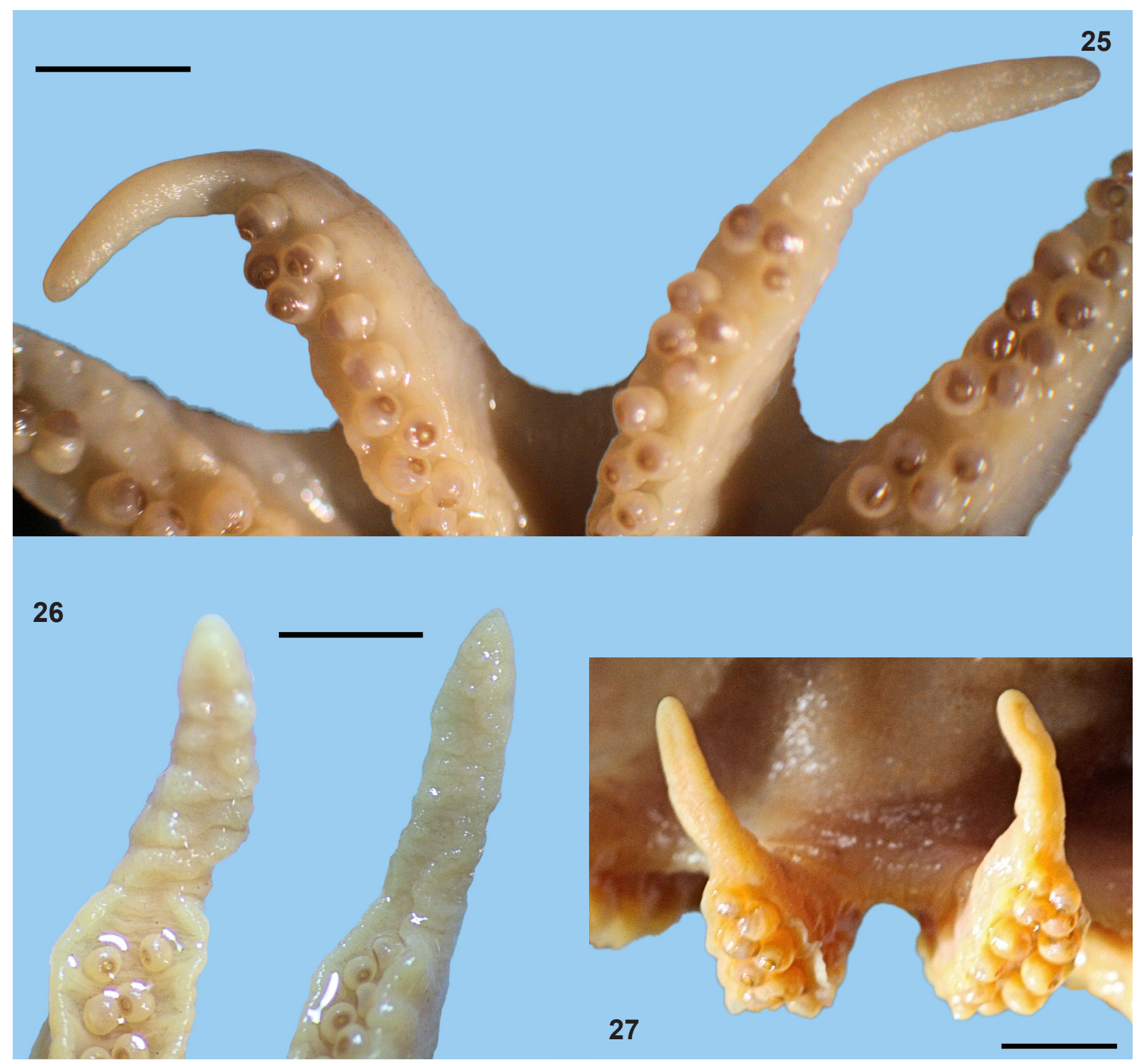

Figs 25-27. Sepia robsoni, tips of I pair arms, various modifications: 25 - SAIAB 209568, male 20 mm ML; 26 - SAIAB 209582, female $26 \mathrm{~mm} \mathrm{ML;} 27$ - SAIAB 209575, male $23 \mathrm{~mm}$ ML. Scale bars $2 \mathrm{~mm}$

starts the fold; ventral sucker starts the next fold and so on) (Fig. 28). Right ventral arm also modified (Fig. 30), sucker arrangement from base to tip: single very large sucker, then eight pairs of greatly enlarged bi-serial suckers; seven pairs of much smaller suckers, gradually becoming smaller; 7-8 pairs of tiny bi-serial suckers to tip.

Tentacular stalk moderately long (85-150\% ML), club large (15-25\% ML) (Figs 31-32) with sub-equal small suckers in 12-16 transverse rows of 3-4 suckers each. Protective membranes narrow, well separated. Natatory membrane well developed, continuing along tentacular stalk for about one club length. Club axis is a continuation of tentacular stalk (Figs 31-32).

Beaks small, fragile, of typical sepiid proportions. Upper beak (Figs 33-34): rostrum blunt, relatively short, slightly hooked, somewhat longer than wide, rostrum angle strongly curved; hood high above crest posteriorly; jaw edge straight, jaw angle $<90^{\circ}$; posterior edge of lateral wall curved; only rostrum and hood dark. Lower beak (Figs 35-37): rostrum short, blunt, jaw angle rounded, broad, $<90^{\circ}$, cutting edge straight; hood low on crest; crest curved; crest and lower edge of lateral wall not parallel; shallow fold on lateral wall; posterior edge of lateral wall oblique and rounded; only rostrum, anterior part of hood and anterior part of shoulders dark.

Radula (Fig. 24) simple, homodont, with seven teeth in single row: sharp, pointed, forming high triangle indented at base. Lateral teeth slightly smaller than rhachidian teeth, relatively straight. Marginal teeth long, straight, blunt. 


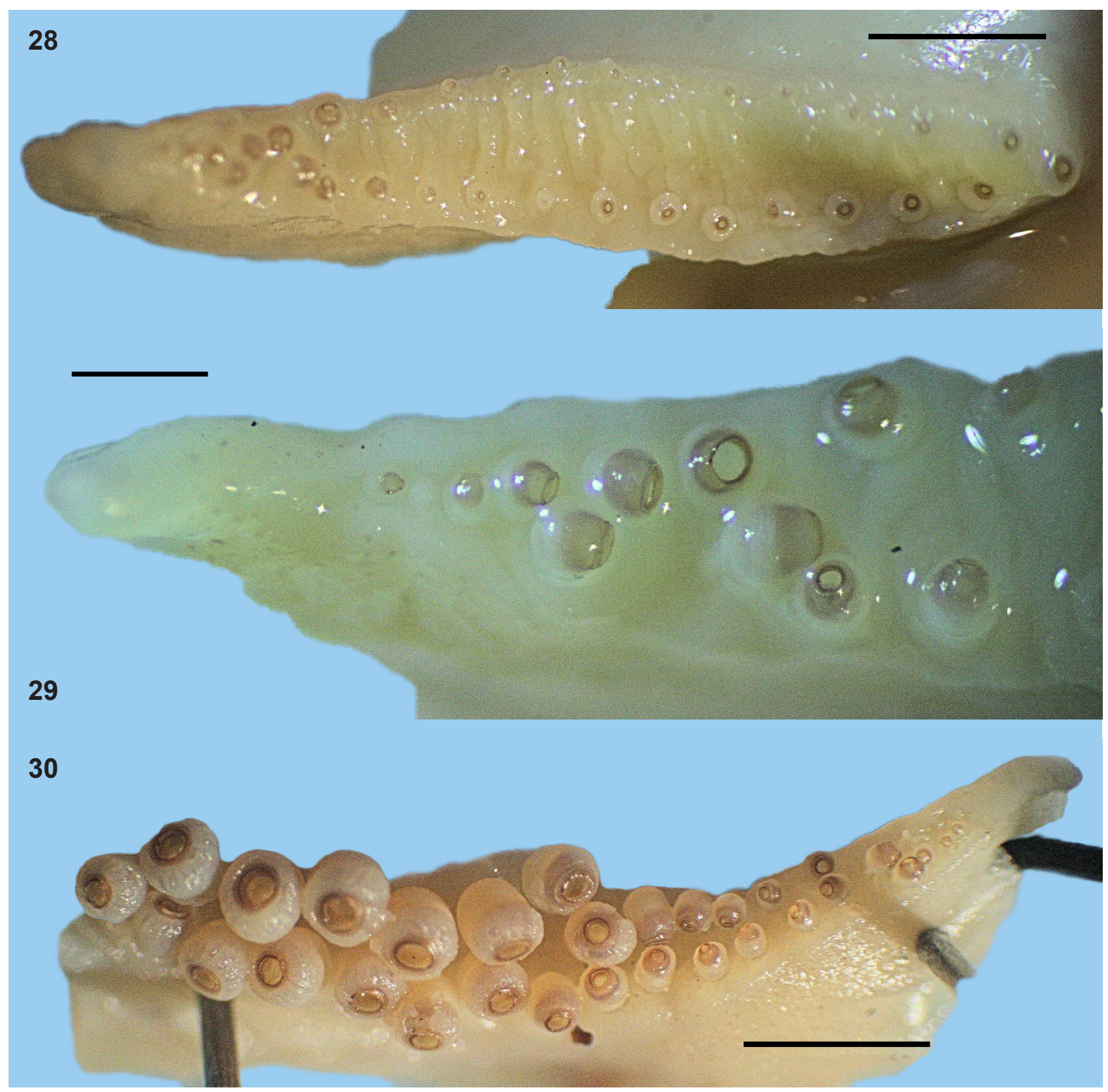

Figs 28-30. Sepia robsoni, tips of I pair arms, various modifications: 28-30 - SAIAB 209568, male 20 mm ML: 28 - hectocotylised left arm IV, 29 - tip of hectocotylised arm IV, 30 - right arm IV. Scale bars 2 mm (28, 30), 0.5 mm (29)

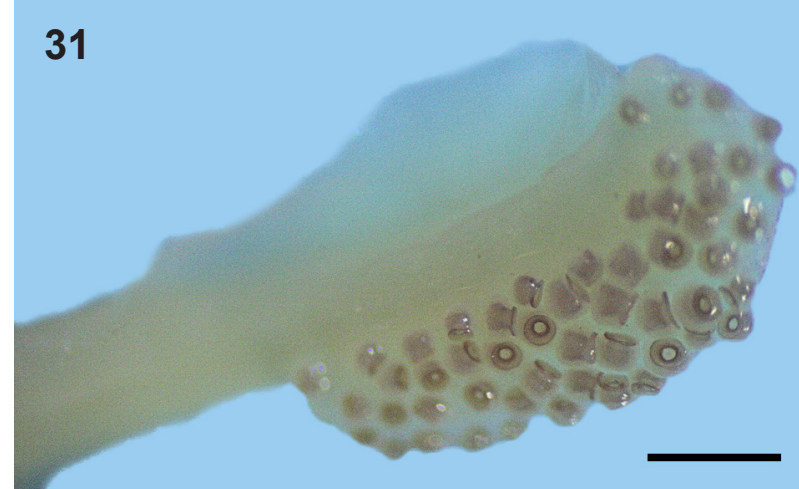

\section{2}

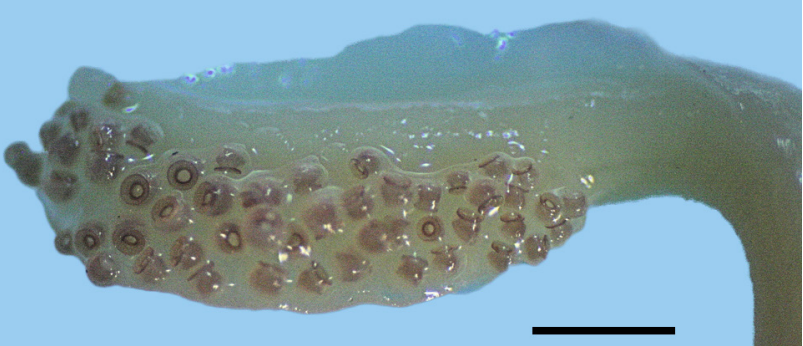

Figs 31-32. Sepia robsoni, clubs, SAIAB 209568, male 20 mm ML: 31 - right; 32 - left. Scale bars 1 mm 
Spermatophores (Fig. 24) seem to bear no species-specific characters, but they are illustrated for the record (for more detailed comparative research in the future).

Locking cartilages: funnel component semi-oval, with internal margin almost straight (Fig. 38), groove deep, simple, without additional median cleft; mantle component simple, prominent (Fig. 39). Nuchal cartilage broad (Fig. 40).

Funnel with valve (Fig. 41). Funnel organ well defined: dorsal part with weak anterior ridge and papilla; arms relatively short, thick and fleshy; ventral part simple, elongated oval (Fig. 41).

Cuttlebone extremely lightly calcified, thin and fragile (Figs $42-47)$. Anterior part ( $25 \%$ of length)
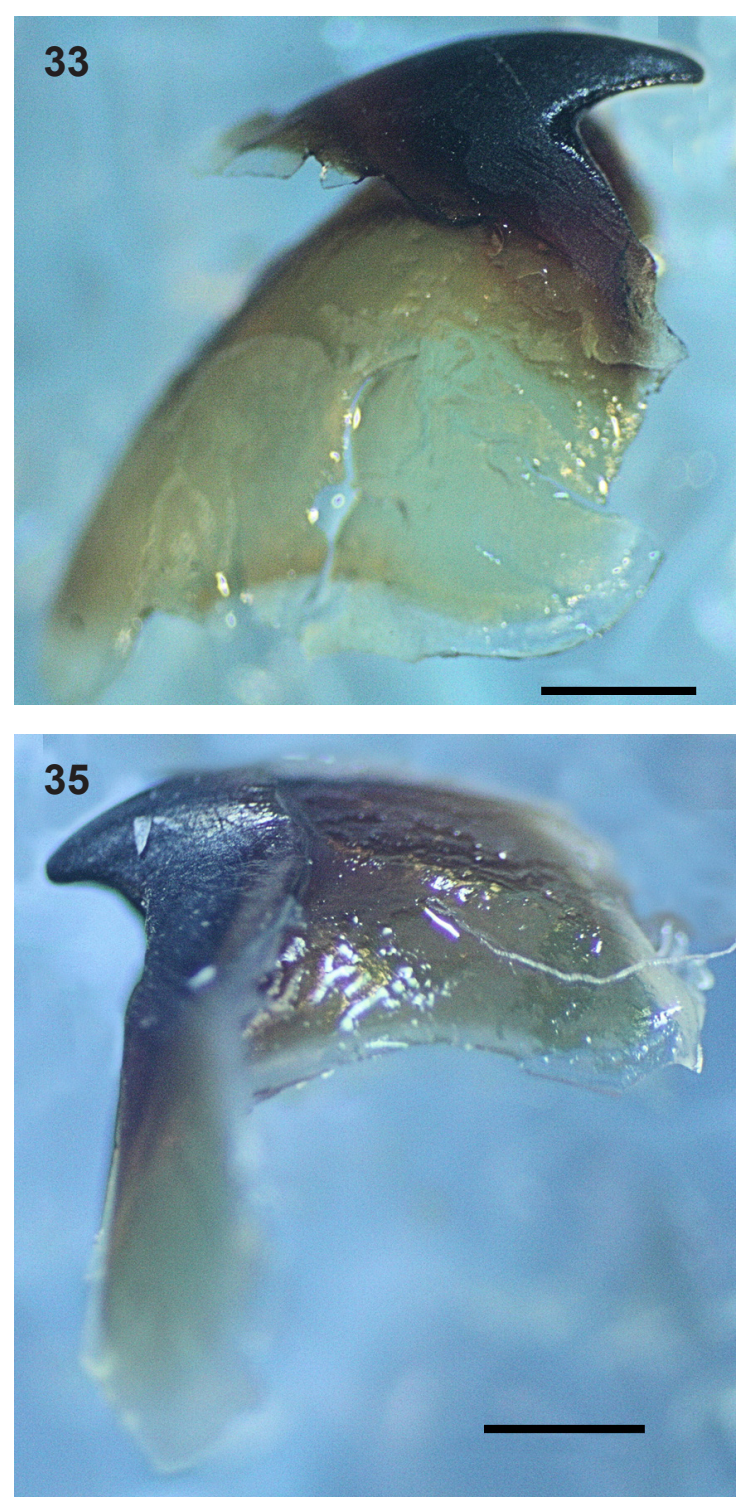

Figs 33-37. Sepia robsoni, beak, SAIAB 209568, male 20 mm ML: 33-34 - upper: 33 - lateral view, 34 - oblique view; 35-37 - lower: 35 - lateral view, 36 - top view, 37 - frontal view. Scale bars $1 \mathrm{~mm}$ triangular, rounded, transparent (formed by dorsal shield only). Middle part occupied by extremely thin, roughly triangular phragmocone (Figs 42, 45) (may
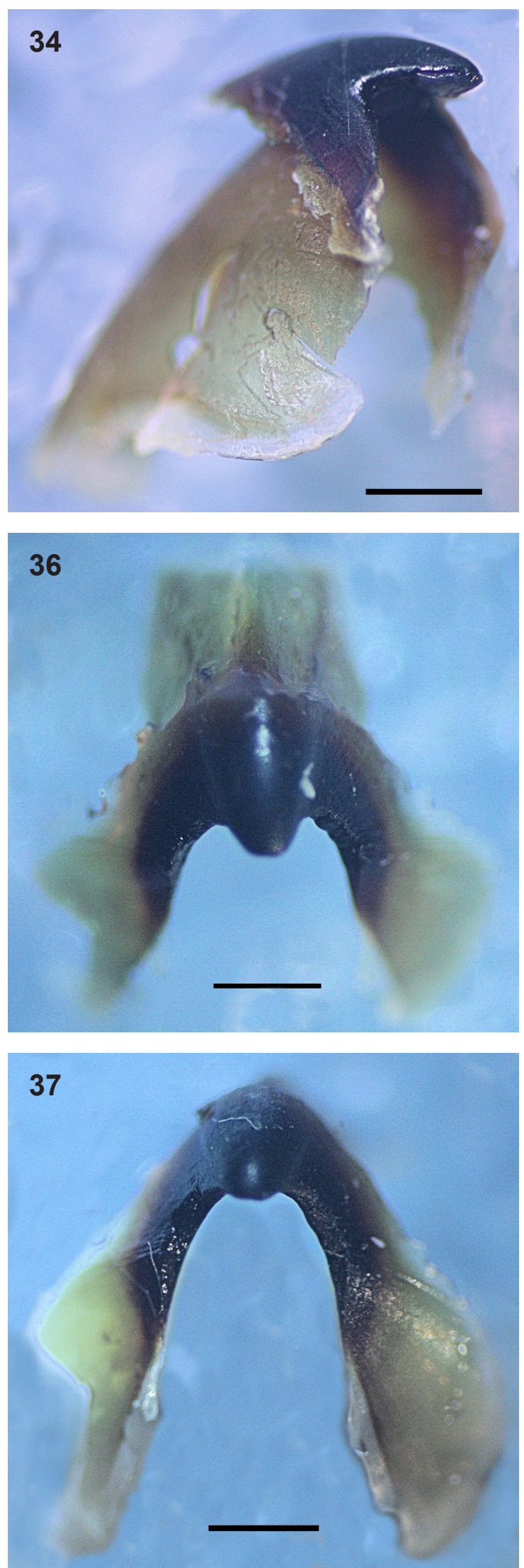


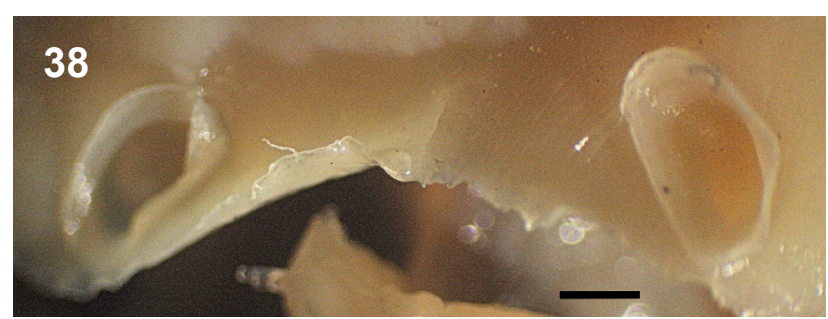

39
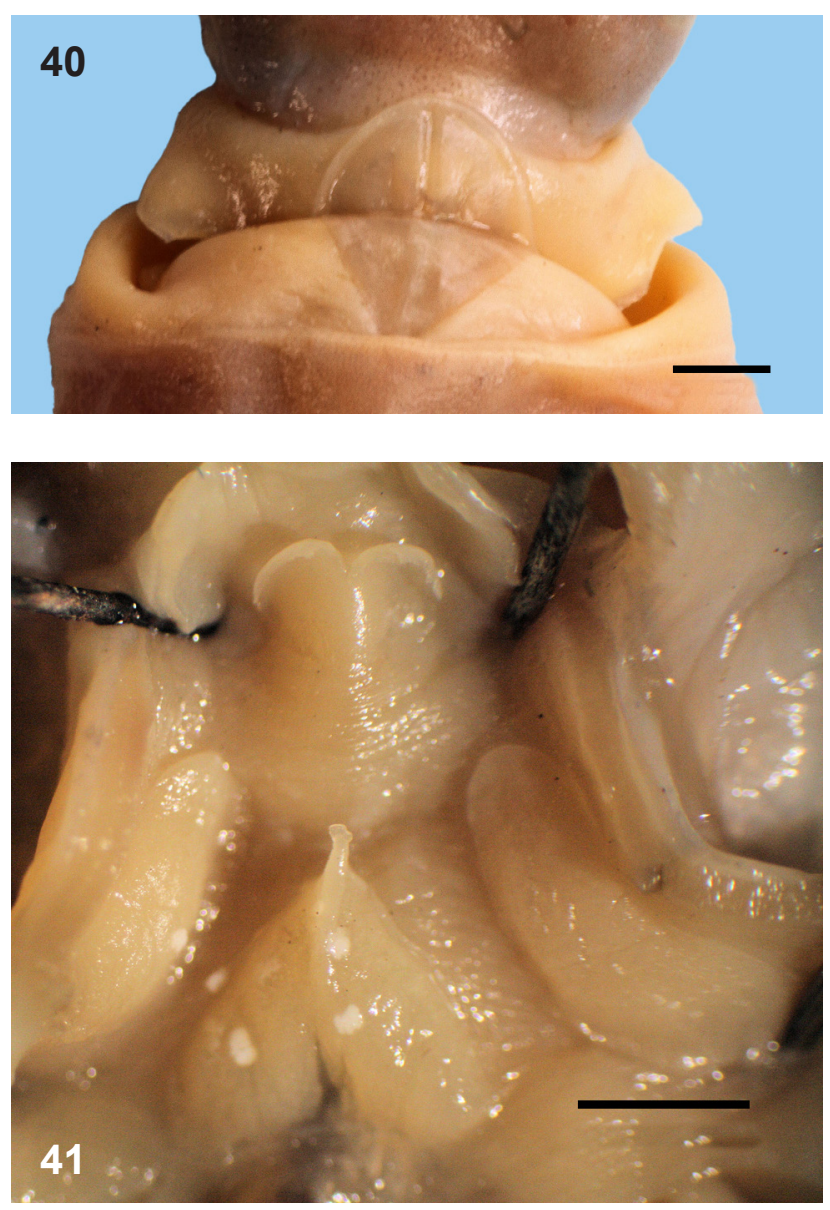

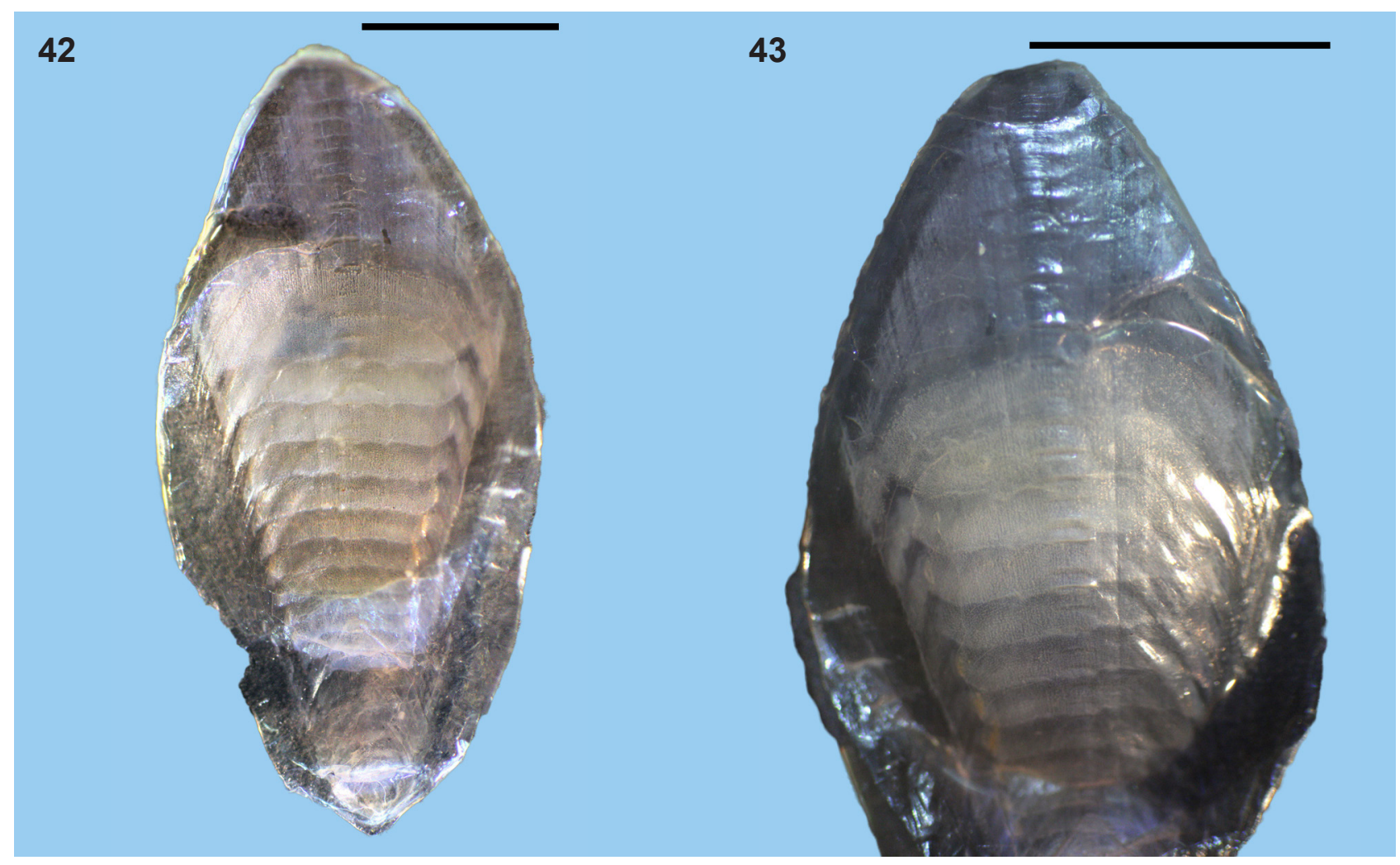

Figs 38-41. Sepia robsoni, SAIAB 209568, male $20 \mathrm{~mm}$ ML: 38-39 - funnel-mantle locking apparatus: 38 - funnel component, 39 - mantle component; 40 - nuchal cartilage, SAIAB 209573, male $18 \mathrm{~mm} \mathrm{ML;} 41$ - funnel organ (dorsal and ventral components) and funnel valve, SAIAB 209568, male $20 \mathrm{~mm}$ ML. Scale bars $1 \mathrm{~mm}$ (3839), $2 \mathrm{~mm}(40-41)$

Figs 42-43. Sepia robsoni, cuttlebone: 42-43 - SAIAB 209581, female $20 \mathrm{~mm}$ ML (preserved in weak ethyl alcohol, damage minimised): 42 - ventral view, 43 - anterior part, dorsal view. Scale bars $5 \mathrm{~mm}$ 

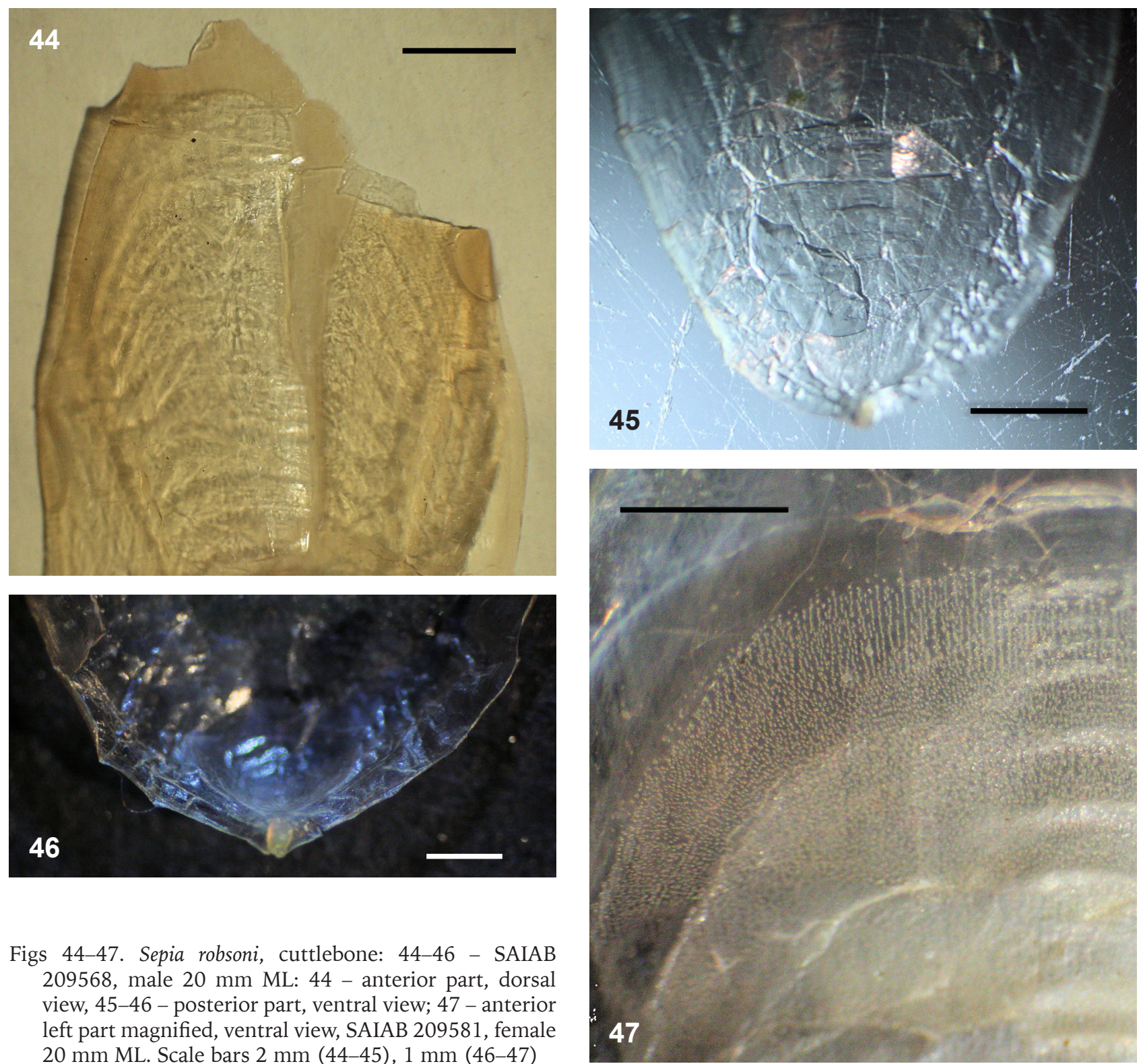

Figs 44-47. Sepia robsoni, cuttlebone: 44-46 - SAIAB 209568, male $20 \mathrm{~mm}$ ML: 44 - anterior part, dorsal view, 45-46 - posterior part, ventral view; 47 - anterior left part magnified, ventral view, SAIAB 209581, female $20 \mathrm{~mm}$ ML. Scale bars $2 \mathrm{~mm}$ (44-45), $1 \mathrm{~mm}$ (46-47)

be dissolved completely in even slightly acid preservative, leaving only faint marks - Fig. 45). Septa thick, straight in middle, convex at margins, first (anterior) one not thickened, with dot calcification (Fig. 47), far from anterior border. Striae strongly convex. Dorsally, along mid-line, elevated strip which broadens anteriorly, forms low but distinct ridge. Growth layers of dorsal shield visible. At base (anteriorly), inner cone is thin and flat, completely fused with phragmocone and dorsal shield. Forked limbs absent, completely reduced. What appears as small "tubercles" marginally and anteriorly, are signs of dot calcification. Outer cone broad, transparent. Posterior cone transparent, ends in distinct yellow knob (no spine). No sexual dimorphism was observed in cuttlebone characters.

Remarks. In the small size range investigated this small-sized species is sexually mature at $22 \mathrm{~mm} \mathrm{ML}$ (both sexes). Juveniles have not been recorded.

MASSY (1927) stated that among the "grooved" arm suckers "none is enlarged in the male". However, since she examined no females, this statement is not correct (compare Figs 19-22). Considering natural variation, I found no other discrepancies between Massy's description and the present one. I have provided a photograph of the holotype (Appendix: Fig. A1) for comparisons and future reference.

Sepia robsoni differs from all other small sepiids (described so far) in the following characters: absence of ventral pores (present in S. typica); tips of I pair of arms devoid of suckers, which is easily seen despite the small size of the species (normal suckers to the end in S. pulchra Roeleveld et Liltved, 1985, S. shazae Lipiński et Leslie, 2018, S. dubia Adam et Rees, 1966); dorsal mantle, head and arms smooth with no elaborate papillose structures (present in Sepia barosei Lipiński, 2020) or dense tubercles (present in $S$. 
faurei Roeleveld, 1972). There are no dense warts nor large tubercles on the dorsal head (present in S. roeleveldi Lipiński, 2020).

Each of these species seems to differ rather profoundly from all others in the cuttlebone characters, although cuttlebones of small-sized sepiids themselves, as a group, may have distinct characters in common. However, this needs to be further examined, described and illustrated by a separate, comparative study. Cuttlebones of all small-sized sepiids are very difficult to dissect without damage because of their great fragility (LIPIŃSKI 2020).

Distribution. S. robsoni, probably near endemic to South Africa, is known both from western and southern waters of South Africa (Fig. 1). The known depth range is $<37-449 \mathrm{~m}$ (specimens obtained by bottom trawls), therefore their habitat is probably diverse.

\section{Sepia (Digitosepia) faurei Roeleveld, 1972 \\ (Figs 48-87, Table 3)}

Holotype. SAMC Iziko SAM A30144. Adult female, ML $21 \mathrm{~mm}$, TW g. R/V Pieter Faure demersal survey, 19 Feb. 1902, Station P.F. 14290, approximate position $88 \mathrm{~km}$ east of Cape Seal (Plettenberg Bay) at depth 168 m. Examined September 1997.

Table 3. Measurements $(\mathrm{mm})$, weight $(\mathrm{g})$ and counts recorded for characters of the holotype and selected specimens of Sepia faurei

\begin{tabular}{|c|c|c|c|c|c|c|c|}
\hline $\begin{array}{l}\text { Catalogue } \\
\text { number }\end{array}$ & $\begin{array}{c}\text { SAMC } \\
\text { A30144 } \\
\text { Holotype }\end{array}$ & $\begin{array}{c}\text { SAIAB } \\
209586\end{array}$ & $\begin{array}{l}\text { SAIAB } \\
209587\end{array}$ & $\begin{array}{l}\text { SAIAB } \\
209588\end{array}$ & $\begin{array}{l}\text { SAIAB } \\
209589\end{array}$ & $\begin{array}{c}\text { SAMC } \\
\text { A089311 }\end{array}$ & $\begin{array}{c}\text { SAMC } \\
\text { A089325 }\end{array}$ \\
\hline Sex & $\mathrm{F}$ & M & $\mathrm{F}$ & $\mathrm{F}$ & $\mathrm{F}$ & $\mathrm{F}$ & $\mathrm{F}$ \\
\hline Maturity & $\mathrm{V}$ & $\mathrm{V}$ & IV & $\mathrm{V}$ & V & IV & $\mathrm{V}$ \\
\hline Weight & 1.43 & 1.02 & 1.77 & 1.91 & 2.44 & 2.52 & 4.81 \\
\hline ML & 21 & 15 & 17 & 20 & 21 & 18 & 29 \\
\hline MLv & 18 & 13 & 15 & 17 & 19 & 17 & 28 \\
\hline $\mathrm{L}$ & $*$ & $*$ & $*$ & 17 & $*$ & $*$ & $*$ \\
\hline WL & $*$ & $*$ & $*$ & 9 & $*$ & $*$ & $*$ \\
\hline HL & 9 & 9 & 9 & 9 & 8 & 7 & 15 \\
\hline HW & 9 & 8 & 9 & 9 & 9 & 6 & 12 \\
\hline $\mathrm{AMH}$ & 2 & 1 & 1 & 2 & 2 & 0 & 1.5 \\
\hline FL & 19 & 14 & 17 & 17 & 18 & 22 & 27 \\
\hline FW & 1.5 & 2 & 2 & 1.3 & 2 & $*$ & $*$ \\
\hline FIa & 2.5 & 2 & 2.6 & 2.3 & 2.5 & 2.2 & 5 \\
\hline FIp & $*$ & 0.3 & $*$ & $*$ & $*$ & 0.5 & * \\
\hline $\mathrm{FFu}$ & 3 & 4 & 4.5 & 5 & 5 & 5 & 6 \\
\hline FuL & 7 & 7 & 7 & 8 & 11 & 9 & 10 \\
\hline AL1-rt & 8 & 7.5 & 10 & 10 & 12 & * & $*$ \\
\hline AL2-rt & 8 & 5 & 6 & 7 & 8 & $*$ & $*$ \\
\hline AL3-rt & 8 & 5 & 8 & 7 & 8 & * & * \\
\hline AL4-rt & 9 & 6.5 & 7 & 8 & 10 & $*$ & $*$ \\
\hline $\mathrm{HcL}$ & - & 6 & - & - & - & - & $*$ \\
\hline MHL & - & 3 & - & - & - & - & $*$ \\
\hline TIP1AL & 1.4 & 1.1 & 1.3 & 1.7 & 1.6 & $*$ & $*$ \\
\hline $\mathrm{TL}$ & 17 & 21 & 18 & $*$ & 25 & $*$ & $*$ \\
\hline $\mathrm{Tcl}$ & 2 & 2 & 2.3 & $*$ & 2.5 & $*$ & $*$ \\
\hline AS1-rt & 0.5 & 0.5 & 0.5 & 0.5 & 0.5 & $*$ & $*$ \\
\hline AS2-rt & 0.5 & 0.5 & 0.5 & 0.5 & 0.5 & * & * \\
\hline AS3-rt & 0.5 & 0.5 & 0.5 & 0.5 & 0.5 & $*$ & $*$ \\
\hline AS4-rt & 0.5 & 0.65 & 0.5 & 0.5 & 0.4 & $*$ & $*$ \\
\hline ASl4 & - & 0.4 & - & - & - & - & $*$ \\
\hline ASl4m & - & 0.25 & - & - & - & - & $*$ \\
\hline $\mathrm{ClS}$ & 0.2 & 0.15 & 0.2 & $*$ & 0.2 & $*$ & $*$ \\
\hline ASC1-rt & $*$ & 25 & 25 & 22 & $*$ & $*$ & $*$ \\
\hline ASC2-rt & $*$ & 28 & 39 & 30 & * & $*$ & $*$ \\
\hline ASC3-rt & $*$ & 28 & 41 & 30 & $*$ & * & $*$ \\
\hline ASC4-rt & $*$ & 38 & 33 & 32 & $*$ & $*$ & $*$ \\
\hline CS\# & 32 & 29 & 32 & $*$ & 32 & * & $*$ \\
\hline CTR\# & 7 & 10 & 8 & $*$ & 10 & $*$ & $*$ \\
\hline CES & 19 & 20 & 21 & $*$ & 22 & $*$ & $*$ \\
\hline CIRC & 4 & 4 & 4 & * & 4 & $*$ & $*$ \\
\hline LORC & 9 & 8 & 9 & $*$ & 12 & $*$ & $*$ \\
\hline
\end{tabular}

- not applicable.

* - missing or impossible to measure.

Holotype was remeasured. 


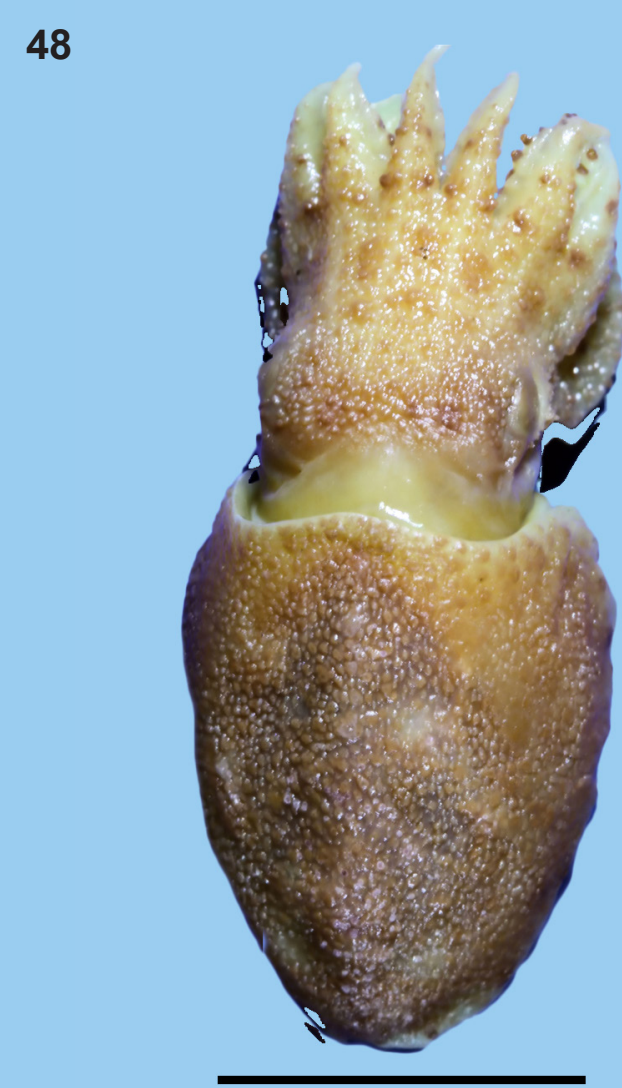

49

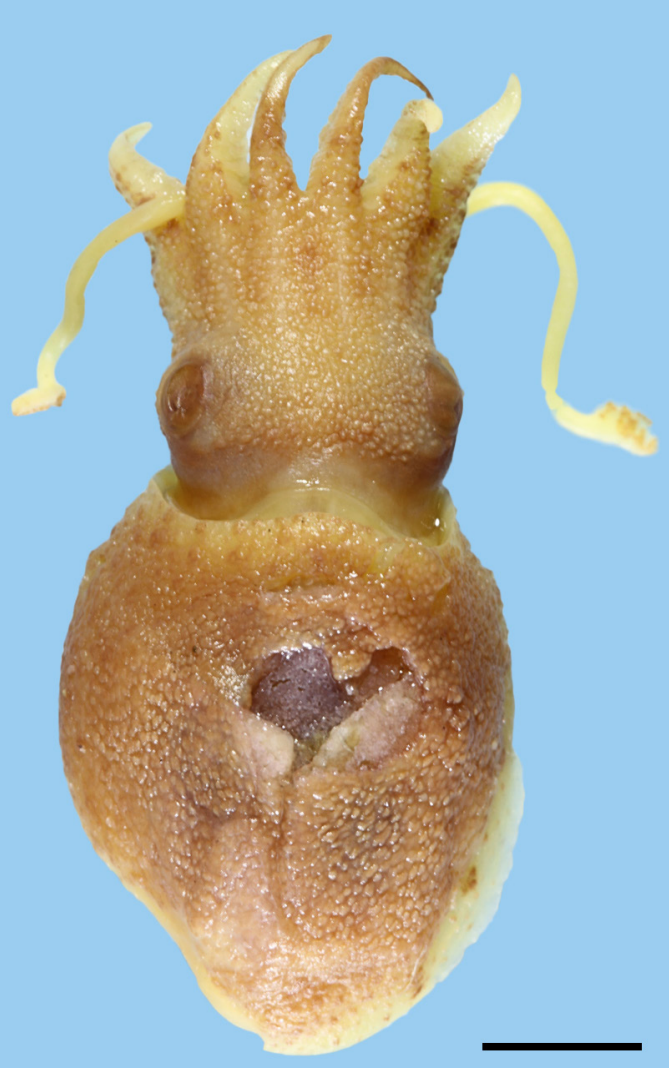

50

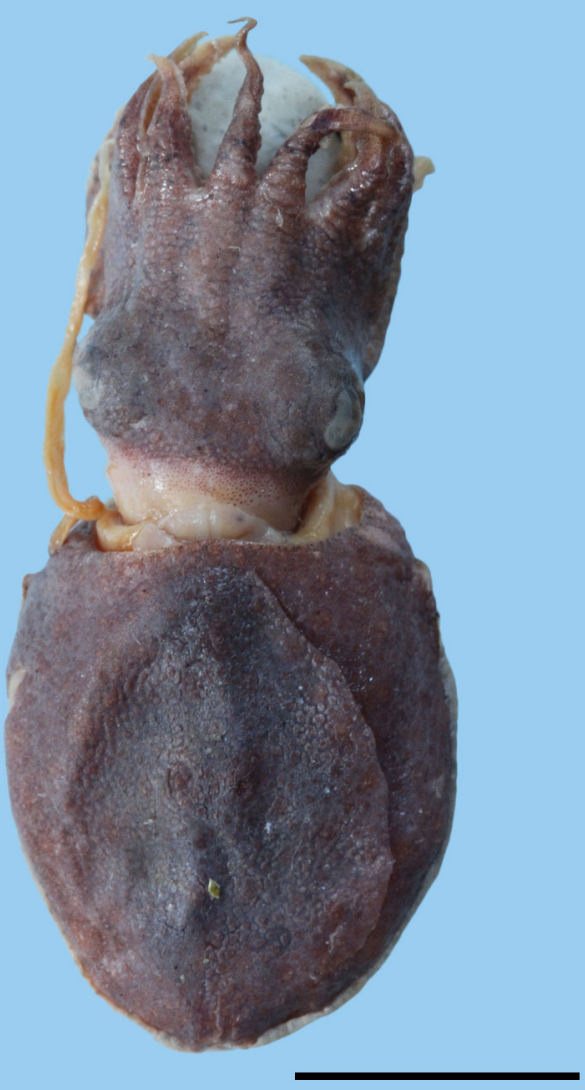

51

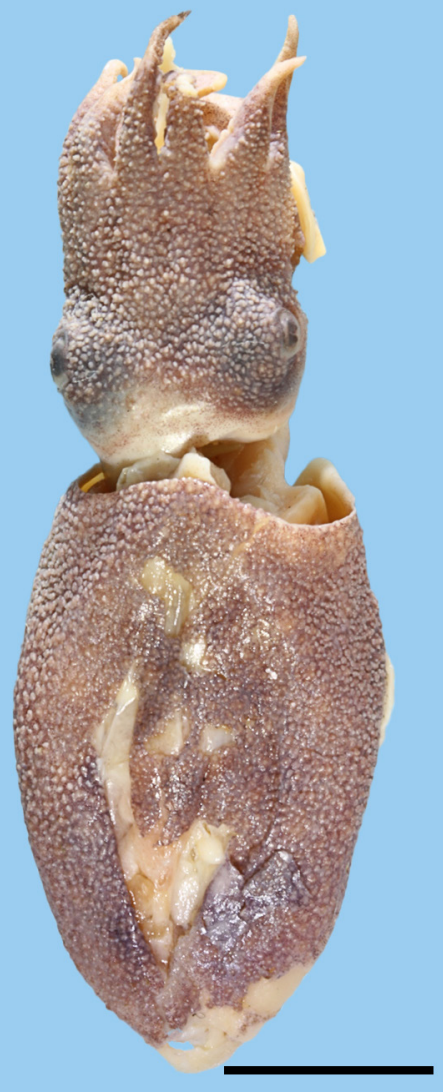

Figs 48-51. Sepia faurei, dorsal view: 48 - SAIAB 209586, male 15 mm ML; 49 - SAIAB 209587, female 17 mm ML; 50 SAMC MB A089311, female 18 mm ML; 51 - SAMC MB A089325, female 29 mm ML. Scale bars 10 mm (48, 50-51), 5 mm (49) 


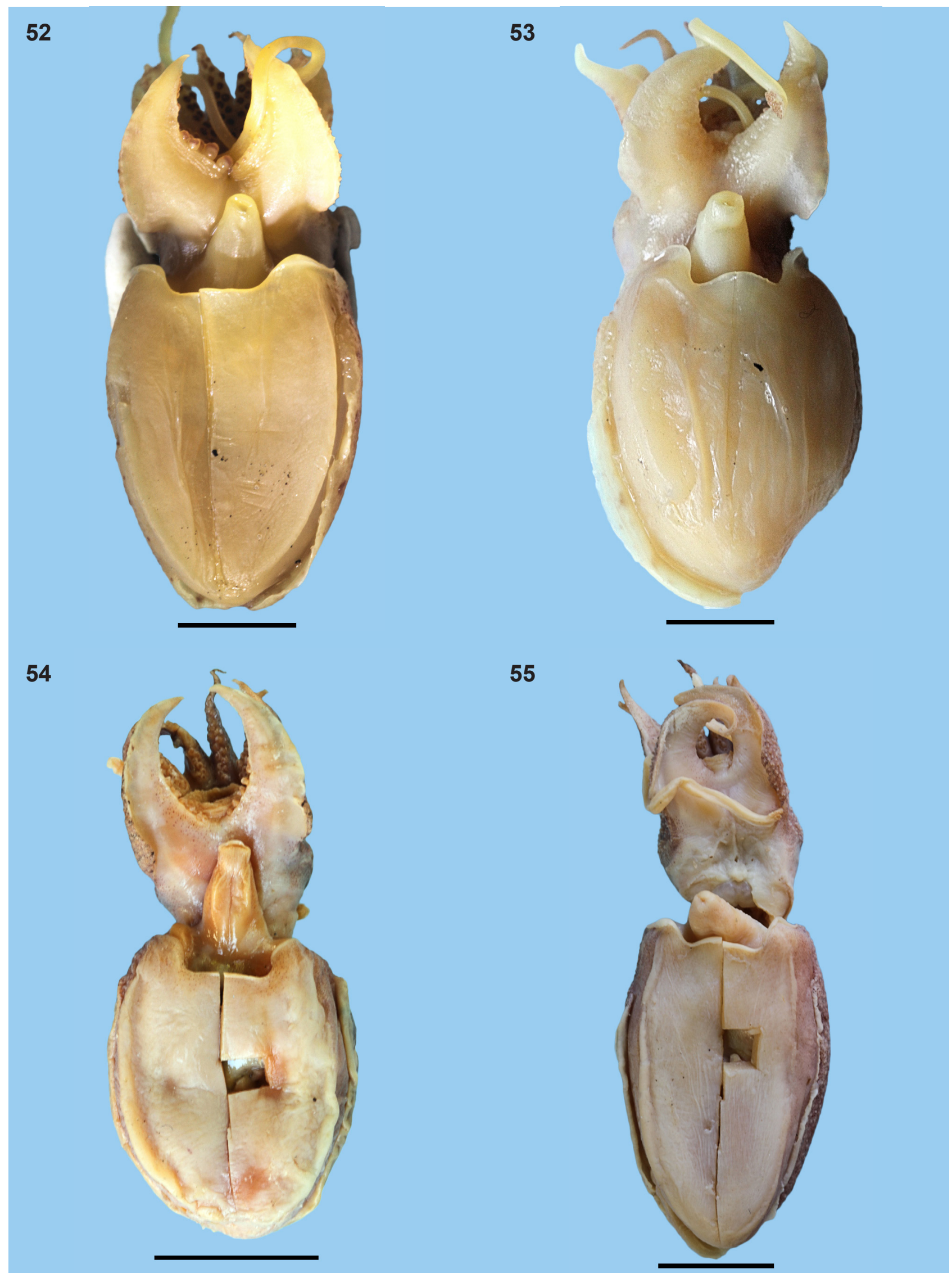

Figs 52-55. Sepia faurei, ventral view: 52 - SAIAB 209586, male 15 mm ML; 53 - SAIAB 209587, female 17 mm ML; 54 SAMC MB A089311, female 18 mm ML; 55 - SAMC MB A089325, female 29 mm ML. Scale bars 5 mm (52-53), 10 mm (54-55) 


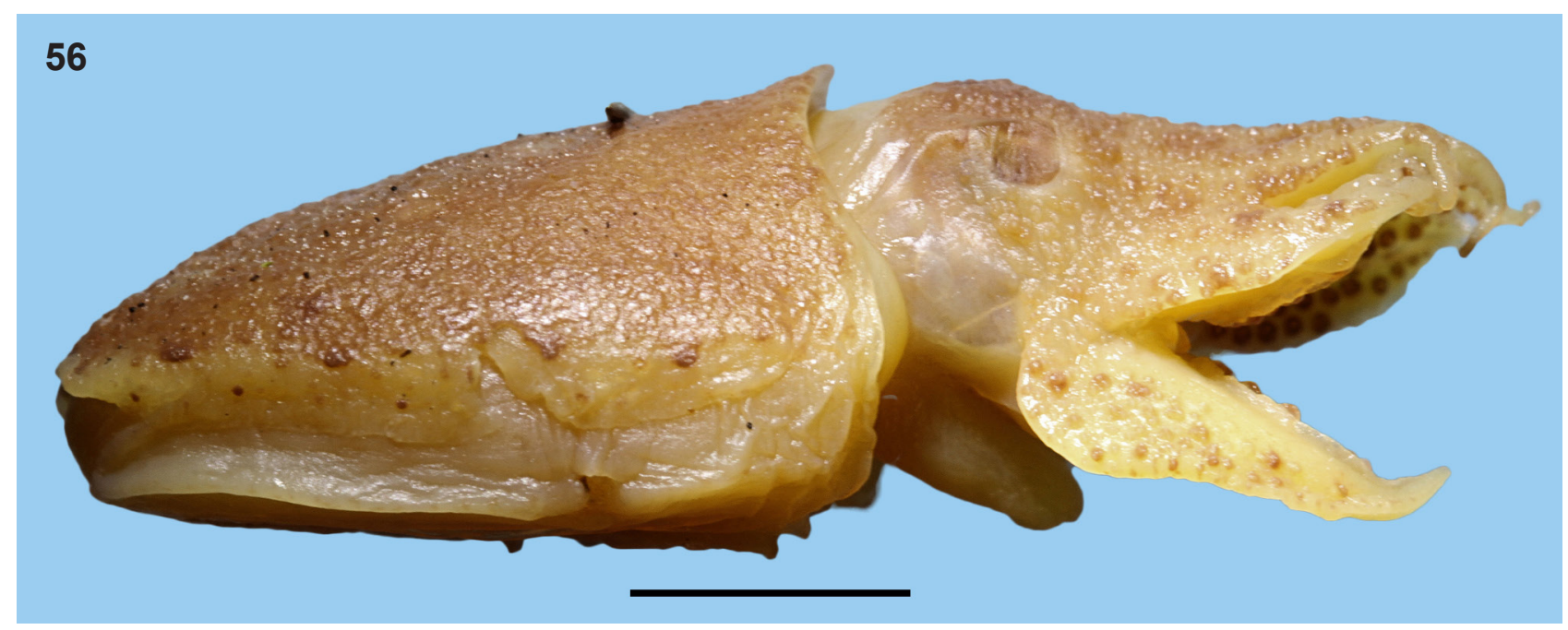

Fig. 56. Sepia faurei, lateral view, SAIAB 209586, male $15 \mathrm{~mm}$ ML. Scale bar $5 \mathrm{~mm}$
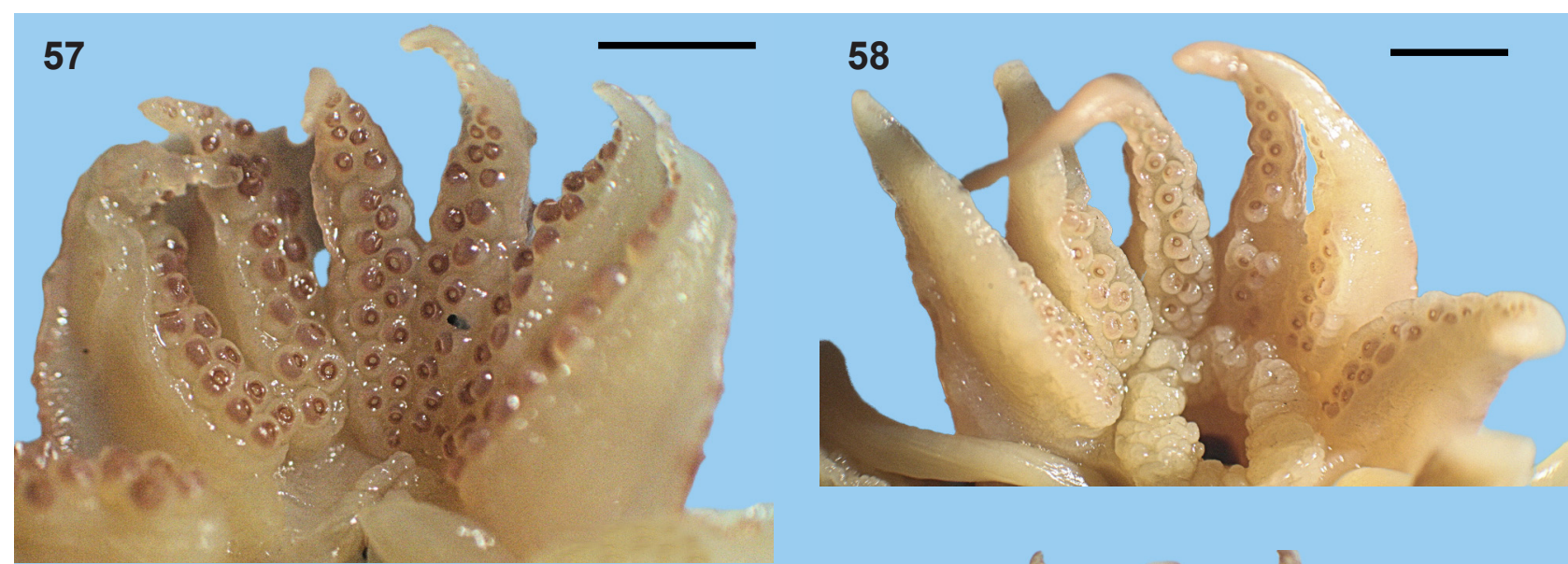

Figs 57-59. Sepia faurei, arms and suckers: 57 - SAIAB 209586, male $15 \mathrm{~mm}$ ML; 58 -SAIAB 209587, female $17 \mathrm{~mm} \mathrm{ML} ; 59$ - inter-brachial web, SAIAB 209588, mature female $20 \mathrm{~mm}$ ML. Scale bars $2 \mathrm{~mm}$

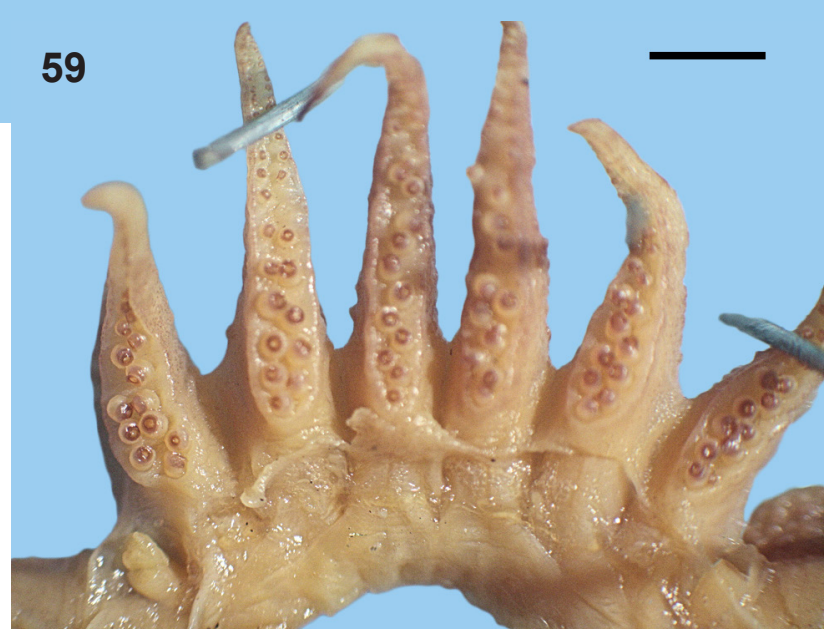

Material. SAIAB 209586. Mature male $15 \mathrm{~mm} \mathrm{ML,}$ TW 1.02 g. R/V Dr Fridtjof Nansen demersal survey, 28 May 2000, AN0182-030-3606, $34^{\circ} 18^{\prime} \mathrm{S}, 24^{\circ} 51^{\prime} \mathrm{E}$, bottom trawl $119 \mathrm{~m}$.

SAIAB 209587. Female $17 \mathrm{~mm}$ ML, TW $1.77 \mathrm{~g}$. R/V Dr Fridtjof Nansen demersal survey, 23 May 2000, AN0161-015-3330, $35^{\circ} 57^{\prime} \mathrm{S}, 21^{\circ} 48^{\prime} \mathrm{E}$, bottom trawl $184 \mathrm{~m}$.

SAIAB 209588. Mature female $20 \mathrm{~mm}$ ML, TW 1.91 g. R/V Dr Fridtjof Nansen demersal survey, 21 May 2000, AN0153-009-3115, 36³'S, 20³1'E, bottom trawl $182 \mathrm{~m}$.

SAIAB 209589. Mature female $21 \mathrm{~mm}$ ML, TW 2.44 g. R/V Dr Fridtjof Nansen demersal survey, 11 Jan. 2010, trawl $16,35^{\circ} 57.5^{\prime} \mathrm{S}, 20^{\circ} 43.8^{\prime} \mathrm{E}$, bottom trawl $139 \mathrm{~m}$. 

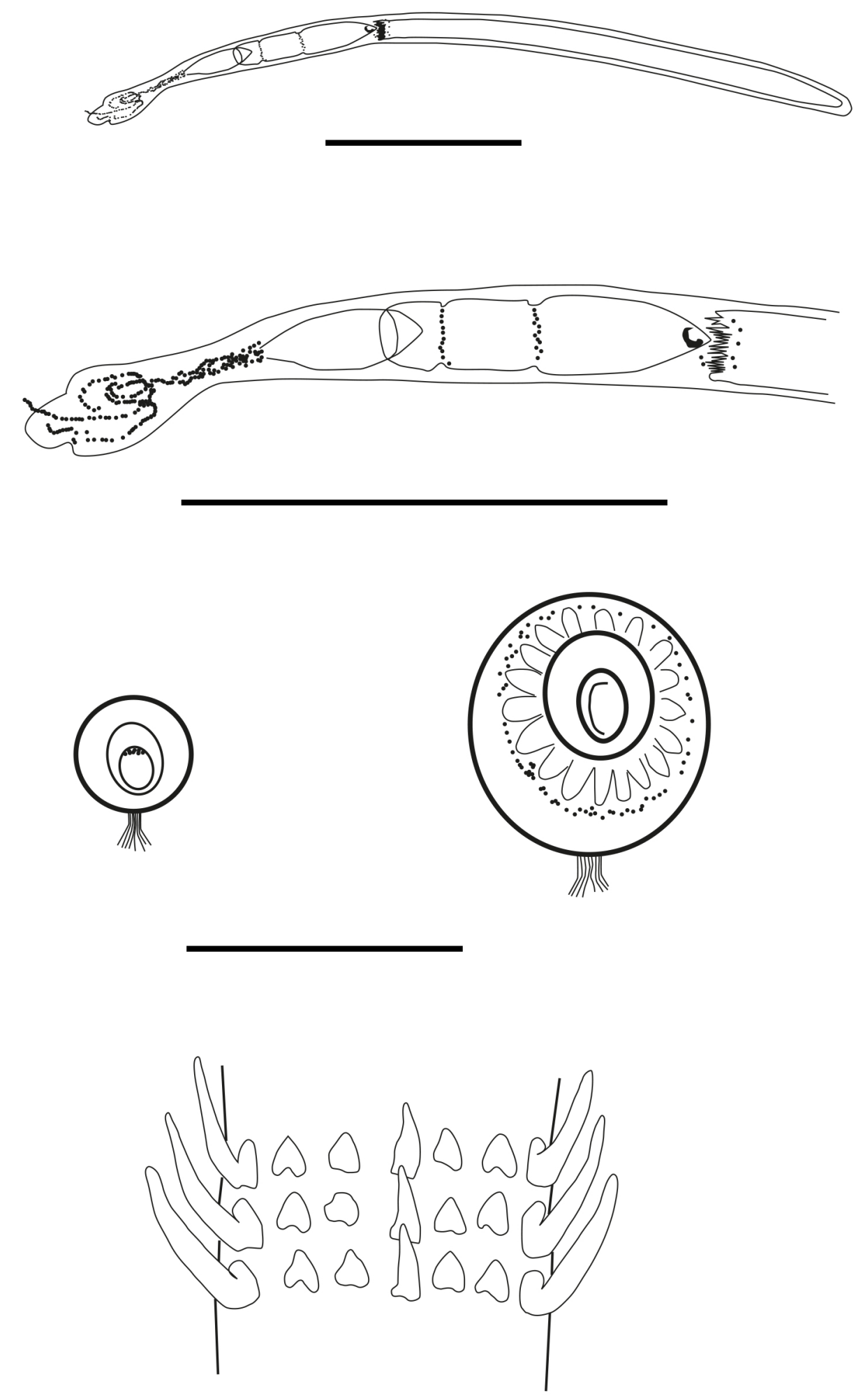

Fig. 60. Sepia faurei, spermatophore and suckers: top - spermatophore, SAIAB 209586, male 15 mm ML; second from top - anterior part of spermatophore magnified; third row (left) - club sucker (middle of central transverse row) and arm IV sucker, 3rd pair from base (suckers viewed from above, all suckers from SAIAB 209587, female $17 \mathrm{~mm} \mathrm{ML);}$ bottom - radula, SAIAB 209587, female $17 \mathrm{~mm}$ ML. Scale bars: $0.5 \mathrm{~mm}$ 
SAMC MB A089311. Female $18 \mathrm{~mm}$ ML, TW 2.52 g. MT Andromeda demersal survey, 17 May 2014, Station D00337-087-3611-86166, $34^{\circ} 17^{\prime} \mathrm{S}, 24^{\circ} 56^{\prime} \mathrm{E}$, bottom trawl $117 \mathrm{~m}$.

SAMC MB A089325. Mature female $29 \mathrm{~mm}$ ML, TW 4.81 g. MT Andromeda demersal survey, 13 Feb. 2015, Station D00381-016-3042-106819, 3607.7'S, $20^{\circ} 10.3^{\prime} \mathrm{E}$, bottom trawl 168-170 m.

SAMC MB A089312. Female $20 \mathrm{~mm}$ ML, TW 1.80 g. MT Compass Challenger demersal survey, 09 May 2016, Station D00773-044-3380-100258, 3531.8'S $22^{\circ}$ 07.4'E, bottom trawl 164-169 m.

Diagnosis. Cuttlebone broad, thick, heavily calcified, not transparent (except outer cone), with posterior (basal) part of inner cone thin and flat, completely fused with outer cone, phragmocone and dorsal shield. Forked limbs absent, completely reduced. Posterior spine absent, but there is inconspicuous dorsal knob. Skin of dorsal mantle and head very densely covered by characteristic tubercles. Thin, long, whip-like tips of I pair of arms devoid of suckers.

Description. Small-sized at maturity; only one male (15 mm ML, mature) and seven females known (1729 mm ML) (Table 3; Figs 48-56). Overall habitus: oval, rather broad (specimens may be elongated or contracted if preserved straight in $96 \%$ ethyl alcohol). Antero-dorsal margin variable: almost straight or very wide "W" type (Figs 48-51); antero-ventral margin emarginated, inverted trapezoid in both sexes, no variation observed (Figs 52-55). Fins robust and wide, ending rather close to anterior mantle margin (FIa 11-17\% ML; Figs 53, 56, Table 3); small gap posteriorly between fins. Colour of dorsal mantle, head and arms in preserved ( $10 \%$ formalin followed by $70 \%$ ethyl alcohol) specimens variable: either reddish brown, dark brown or almost purple (Figs 48-51).

Dorsal mantle, head and arms densely covered with small uniform tubercles (Figs 48-51). Ventral mantle with distinct keels (Figs 52-55). Head robust, neck usually only slightly narrower than head (but see Fig. 50; this may be an effect of long preservation in strong ethyl alcohol). Eyes dorso-lateral (Figs 48-51, 56).

Arms (Figs 57-59; Table 3) II-IV almost subequal in length; arms I longest. However, this was based upon eight individuals only, four of which were measured precisely (other specimens were hardened due to preservation in $96 \%$ ethyl alcohol). Arms relatively short, fleshy; keels especially prominent on ventral (IV) arms; on other arms keel prominence variable (Figs 57-59) and depends upon the state of preservation and possibly other factors. Protective membranes thick, fleshy, suckers bi-serial. Arms connected by membrane; it is the same depth and always much less than $1 / 4$ of arm length between arms of I-III pairs, vestigial between arms III-IV, and

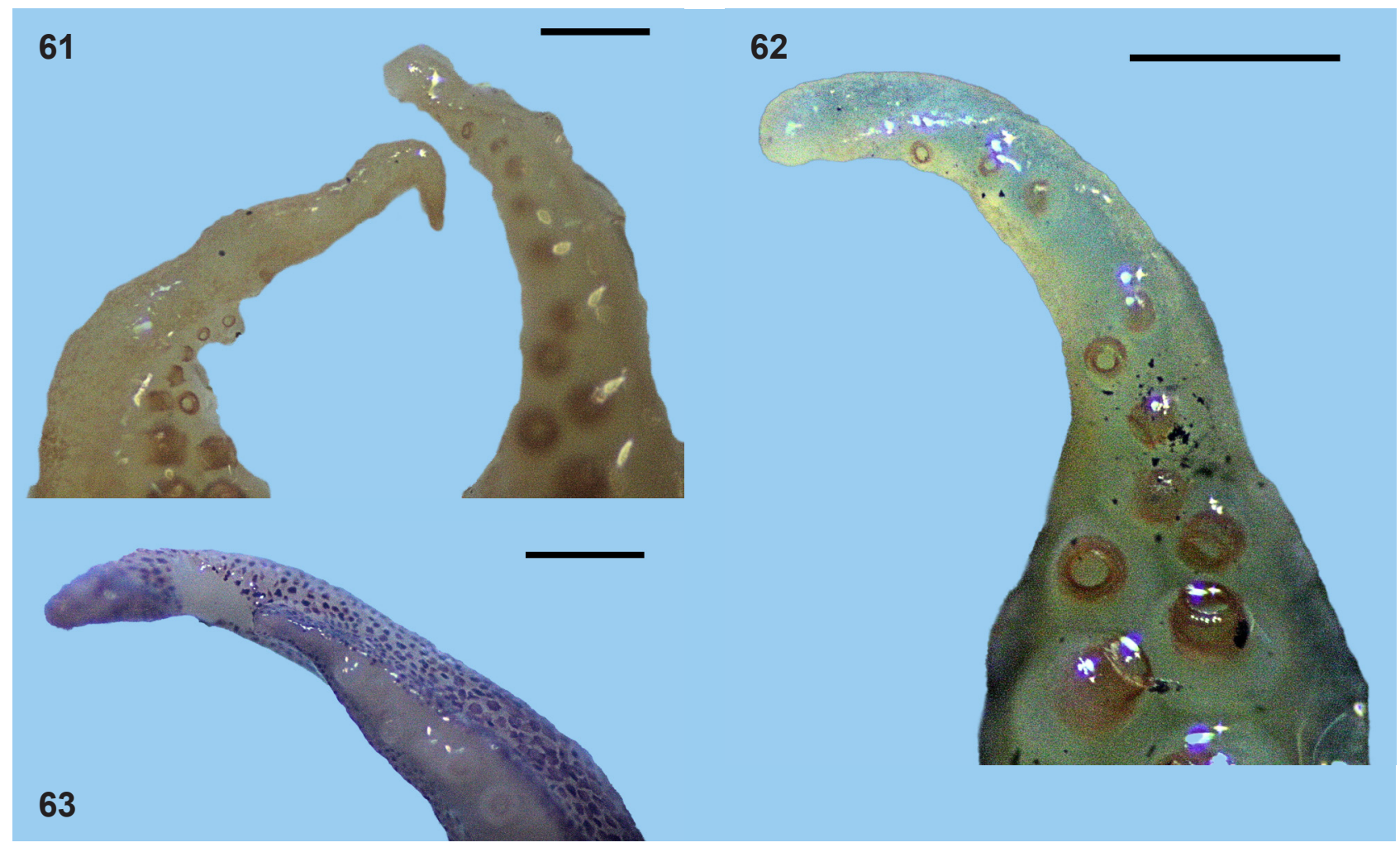

Figs 61-63. Sepia faurei, tips of I pair arms: 61 - SAIAB 209586, male 15 mm ML; 62 - right I pair arm, SAIAB 209586, male 15 mm ML; 63 - left I pair arm, SAIAB 209589, mature female 21 mm ML. Scale bars $1 \mathrm{~mm}$ (61), 0.5 mm (62-63) 
there is no web between arms IV (Fig. 59). Suckers on arms relatively large; sucker rings with no teeth (Fig. 60). Diameter of suckers in females may change abruptly along III and IV arms (after 8th pair and again after 3rd-4th pair), but the character is variable. Tips of arms of I pair generally devoid of suckers (some may bear uni-serial odd suckers); length and structure of arm I tips quite variable; they may be of different length on each arm (range $7-13 \%$ of arm length) (Figs 61-63; Table 3). Left ventral arm hectocotylised (Fig. 64): eight pairs of medium-sized suckers in marginal rows. Suckers of similar size in dorsal and ventral row. Proximally 12 pairs of suckers, decreasing gradually in size towards tip. Low, rather inconspicuous fleshy transverse folds between marginal sucker pairs. Proximal part of hectocotylised arm

64

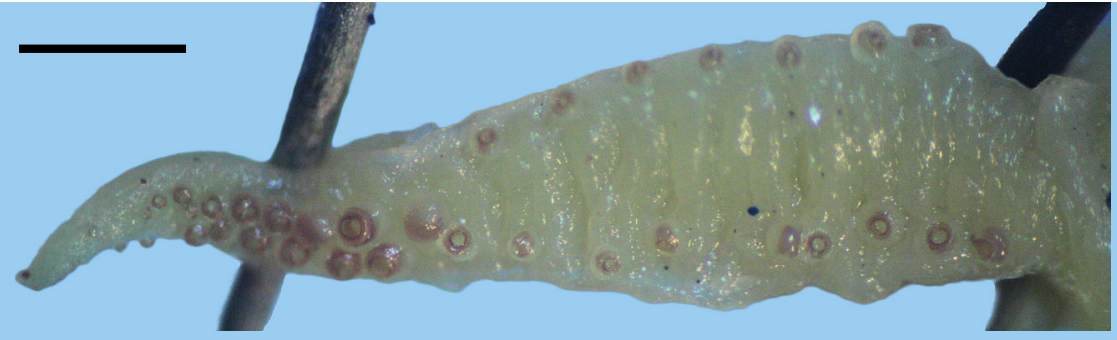

65

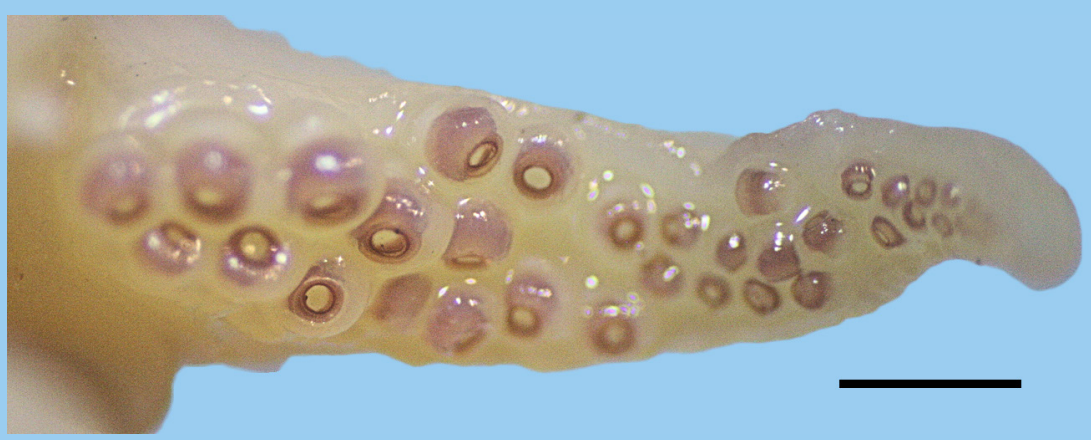

66

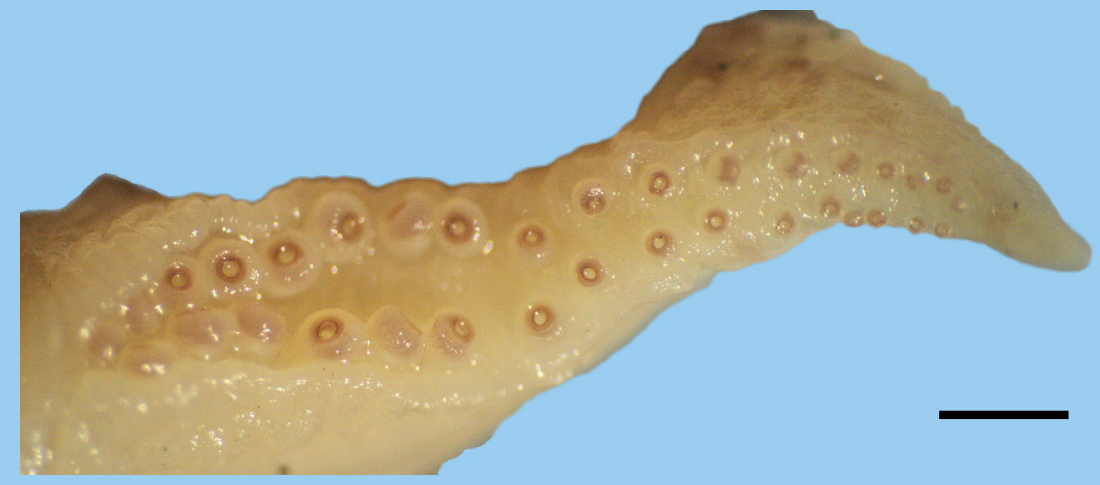

67

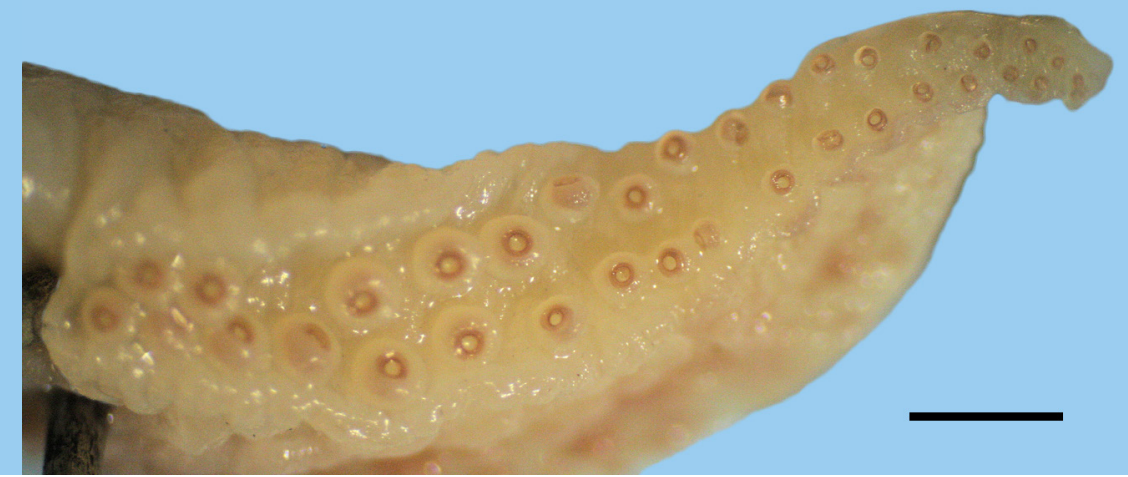

Figs 64-67. Sepia faurei, arms IV: 64 - hectocotylised left arm IV, SAIAB 209586, male 15 mm ML; 65 - right arm IV, SAIAB 209586, male 15 mm ML; 66 - left arm IV, SAIAB 209587, female 17 mm ML; 67 - right arm IV, SAIAB 209587, female $17 \mathrm{~mm}$ ML. Scale bars $1 \mathrm{~mm}$ 
noticeably widened. Right ventral arm not modified (Fig. 65), similar to right IV arm of the female (Fig. 67). Non-hectocotylised arms of males not modified (compare Figs 57-59).

Tentacular stalk of moderate length (106-140\% ML). Club small (12-14\% ML), uniform with 10-11 rows, each with 3-4 suckers (Figs 68-71). Suckers minute (Fig. 60), chitinous rings mostly smooth but tiny indentation seen on some. Protective membranes narrow. Natatory membrane well developed, extends along tentacular stalk for about $1 / 2$ of club length.

Upper beak (Figs 72-73): rostrum blunt, relatively short, not hooked, as long as wide, rostrum angle well defined; hood long, distal tip far from crest, jaw edge straight, jaw angle $90^{\circ}$; lateral wall strongly curved. Only rostrum and hood dark.

Lower beak (Figs 74-76): rostrum short, blunt, lacking distinct rostrum angle; hood low on crest, slightly curved, indented; crest slightly curved, not
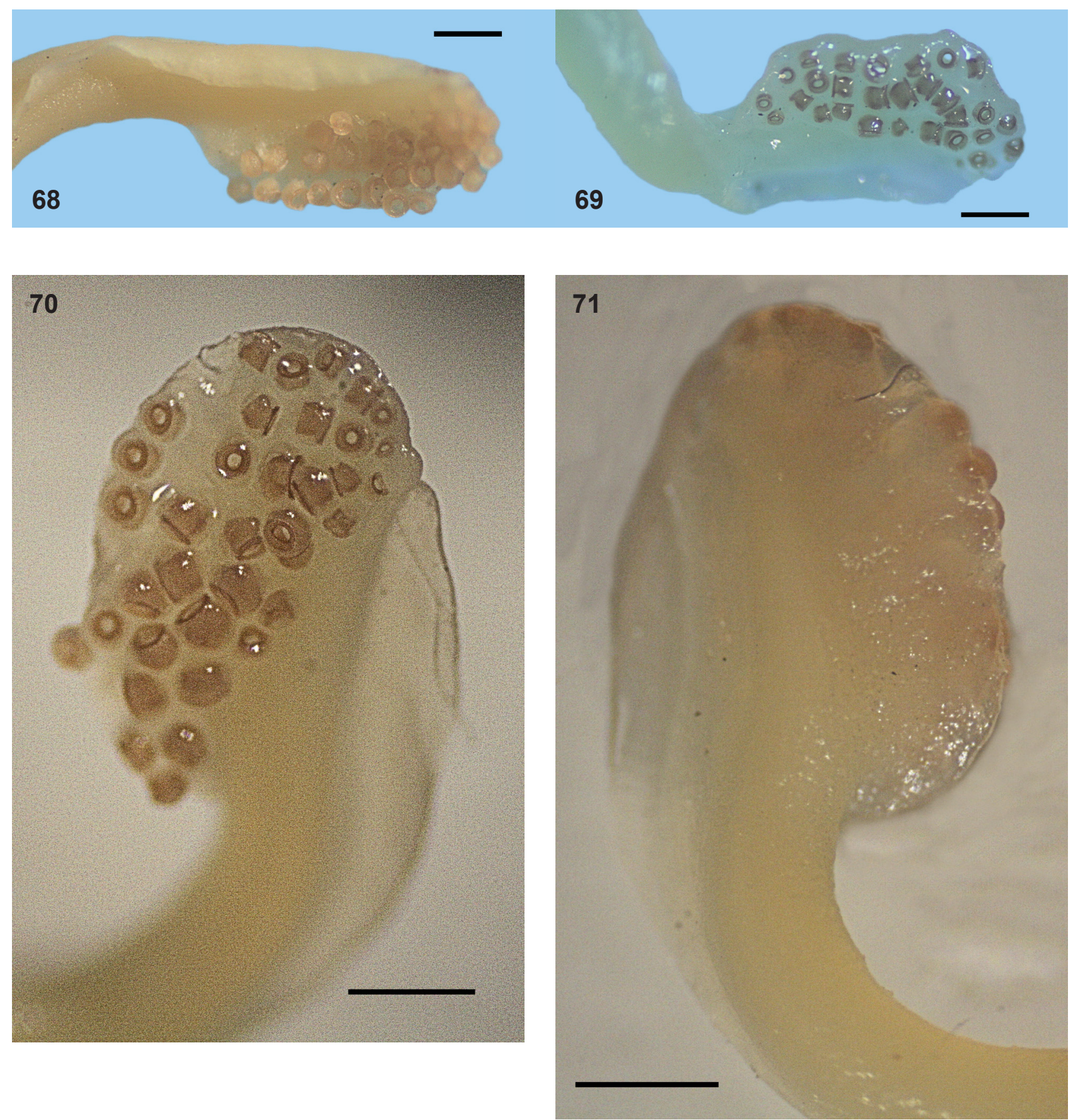

Figs 68-71. Sepia faurei, clubs: 68-69 - SAIAB 209586, male 15 mm ML: 68 - left, 69 - right; 70-71 - SAIAB 209587, female $17 \mathrm{~mm} \mathrm{M:} 70$ - right (ventral view), 71 - right (dorsal view). Scale bars $0.5 \mathrm{~mm}$ 

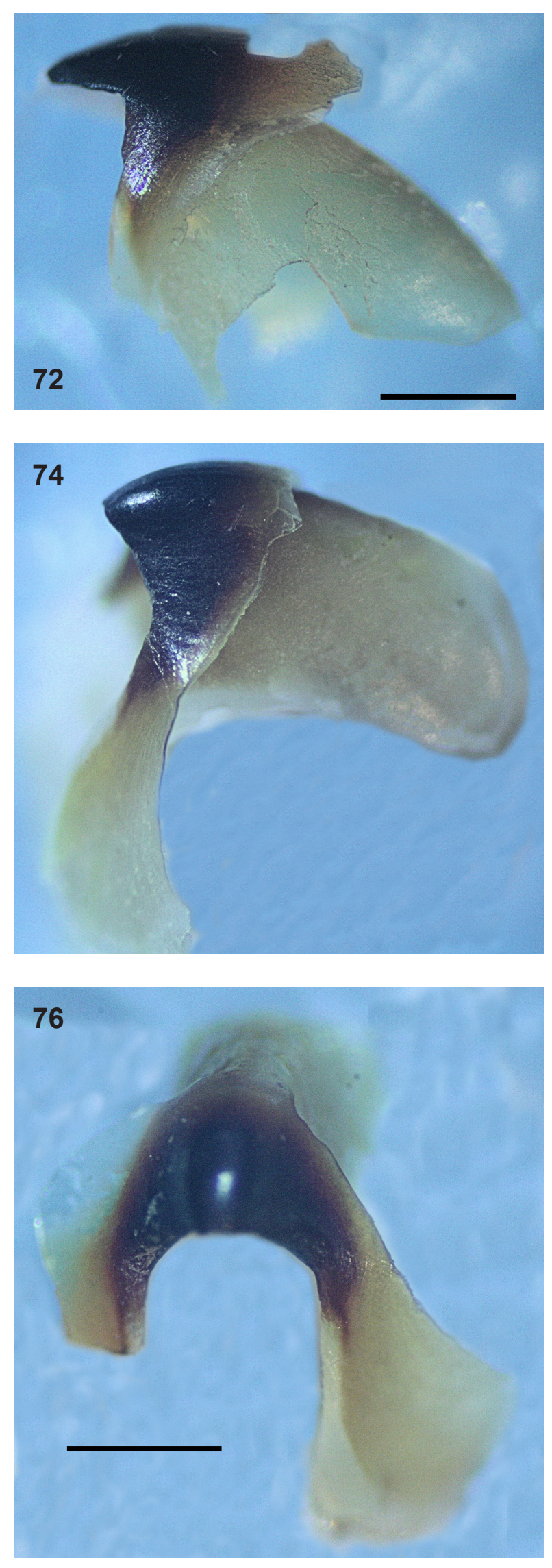
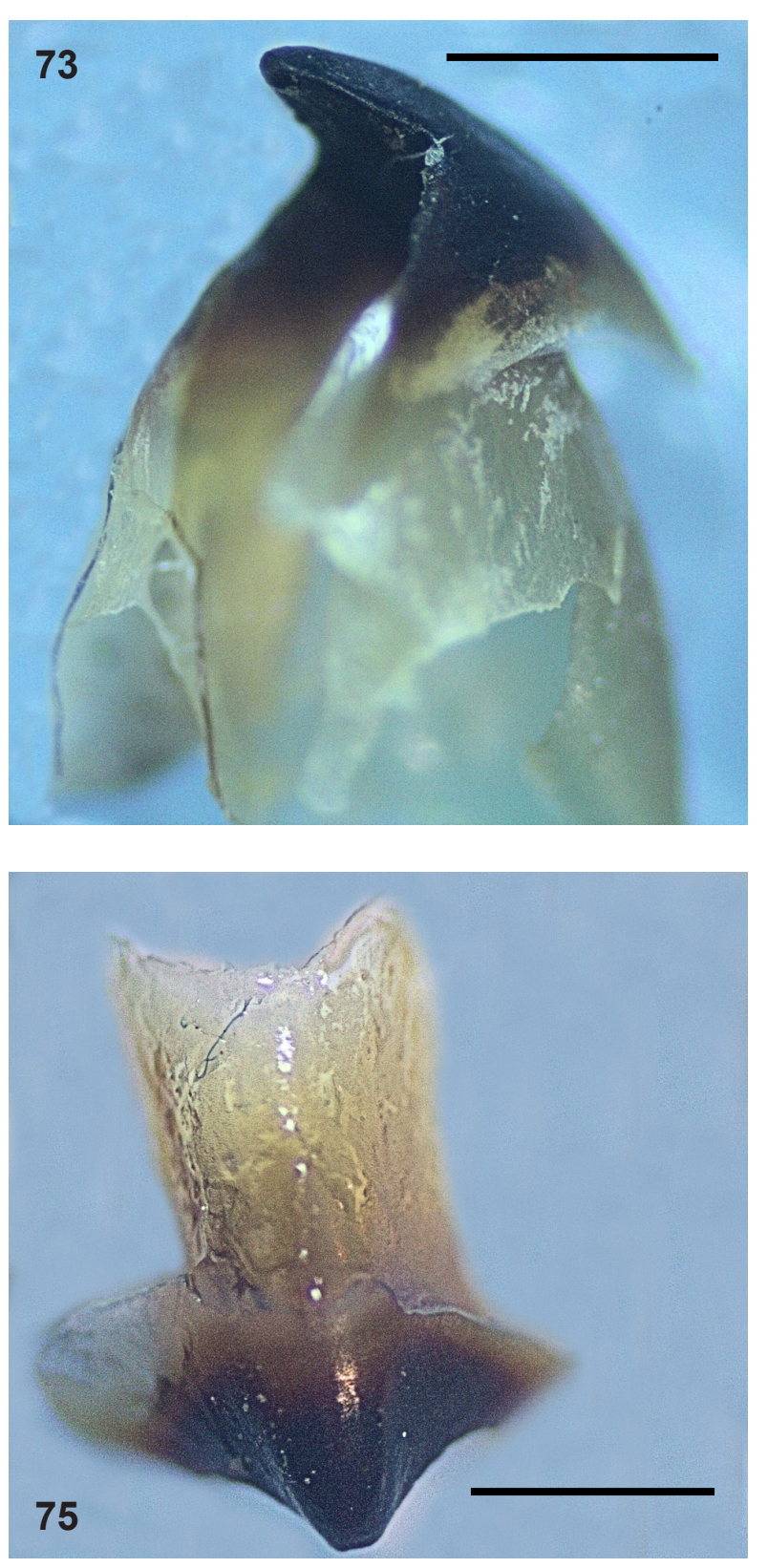

Figs 72-76. Sepia faurei, beak, SAIAB209589, female $21 \mathrm{~mm}$ ML: 72-73 - upper: 72 - letral view, 73 oblique view; 74-76 - lower: 74 - lateral view, 75 - top view, 76 - frontal view. Scale bars $1 \mathrm{~mm}$ 


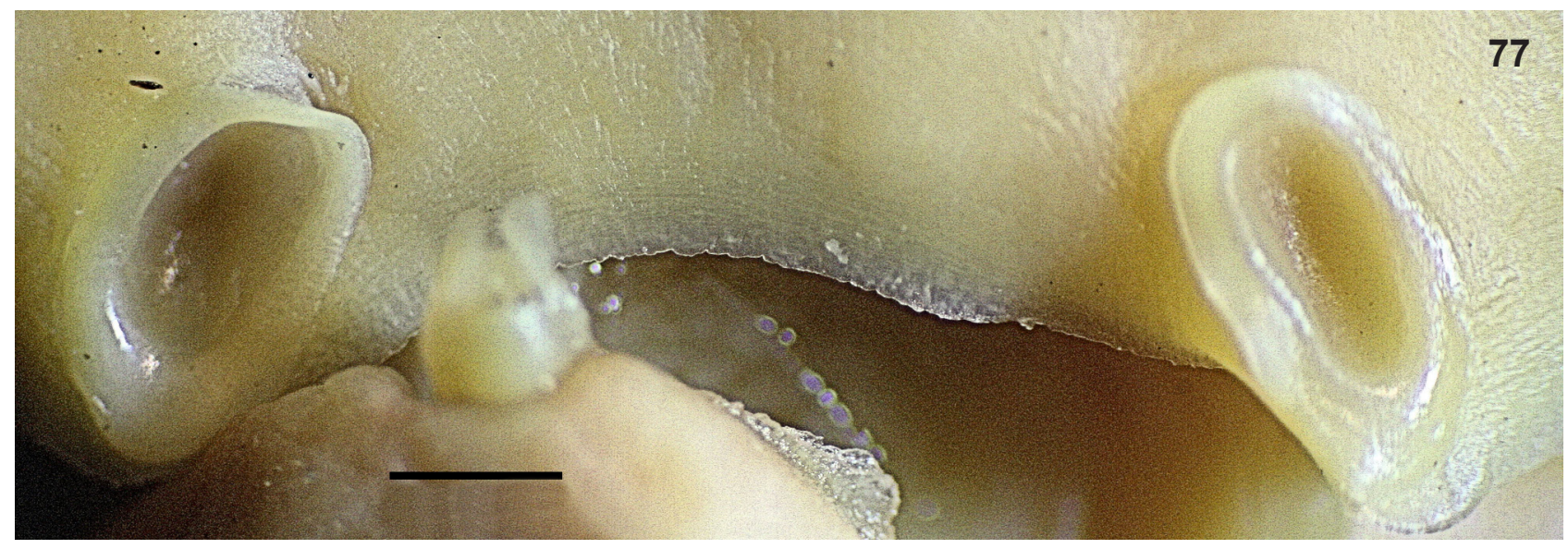

Figs 77-78. Sepia faurei, funnel-mantle locking apparatus, SAIAB 209589, female 21 mm ML: 77 - funnel component; 78 - mantle component. Scale bars $1 \mathrm{~mm}$

indented, not parallel to lower edge of lateral wall (proximally further apart than distally); lateral wall with curved and angled posterior margin, with no fold on upper part of lateral wall. Only rostrum and upper parts of shoulders dark.

Radula homodont, with seven teeth per row (Fig. 60). Marginal plates not detected. Rhachidian teeth high, narrow, sharp, triangular, with small indentation at base. First and second laterals small, squat, symmetrical, with small indentation at base. Heels small, compact. Marginal teeth fairly uniform, long, blunt, moderately curved, not indented.

Spermatophores (Fig. 60) seem to bear no species-specific characters, but they are illustrated for the record (for more detailed comparative research in the future).

Locking cartilages: funnel components beanshaped (Fig. 77), internal margins straight, groove rather shallow, without additional median cleft; mantle components simple, short (Fig. 78).

Funnel with valve (Fig. 79). Funnel organ with dorsal component well defined, bearing anterior ridge and papilla, limbs short. Ventral component well defined, long and narrow (Figs 80-81).

Cuttlebone (Figs 82-83) broad, thick, heavily calcified, not transparent (except outer cone), with posterior part of inner cone as thin band, completely fused with outer cone, phragmocone and dorsal shield. Forked limbs absent, completely reduced. Outer cone broad, transparent, surrounds phragmocone and continues to anterior extremity. Septa strong, convex, closely spaced, they extend to anterior margin (as in most sepiids). Striae convex, strong. Dorsal shield simple, with no deposits. Posterior spine absent; inconspicuous knob on dorsal side of inner cone. If the cuttlebone is de-calcified during preservation (Figs 84-87), it may undergo secondary,

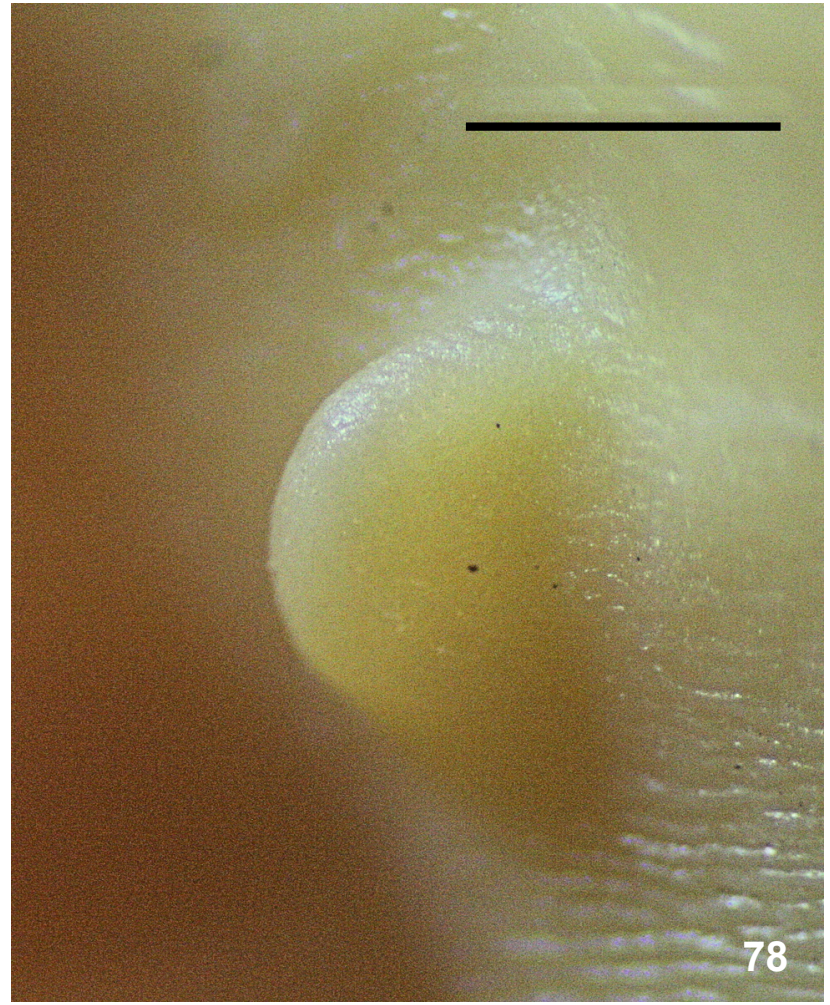

runaway calcification (Figs 84-85) or leave the dorsal shield with remnants of the phragmocone with strongly convex striae (Figs 86-87).

Remarks. Sepia faurei differs distinctly from all other small sepiids in the prominent tubercles, densely covering entire dorsal mantle, head and arms. There are other southern African Sepia species with mantle, head and arms covered by tubercles: Sepia tuberculata Lamarck, 1798, S. papillata Quoy et Gaimard, 1832 and S. simoniana Thiele, 1920. However, in each case these tubercles are different; their detailed morphology and distribution should be examined comparatively, possibly on a worldwide scale. This, as well as the study of the cuttlebone, may prevent many mistakes and misunderstandings when undertaking future descriptions. All mentioned species differ pro- 

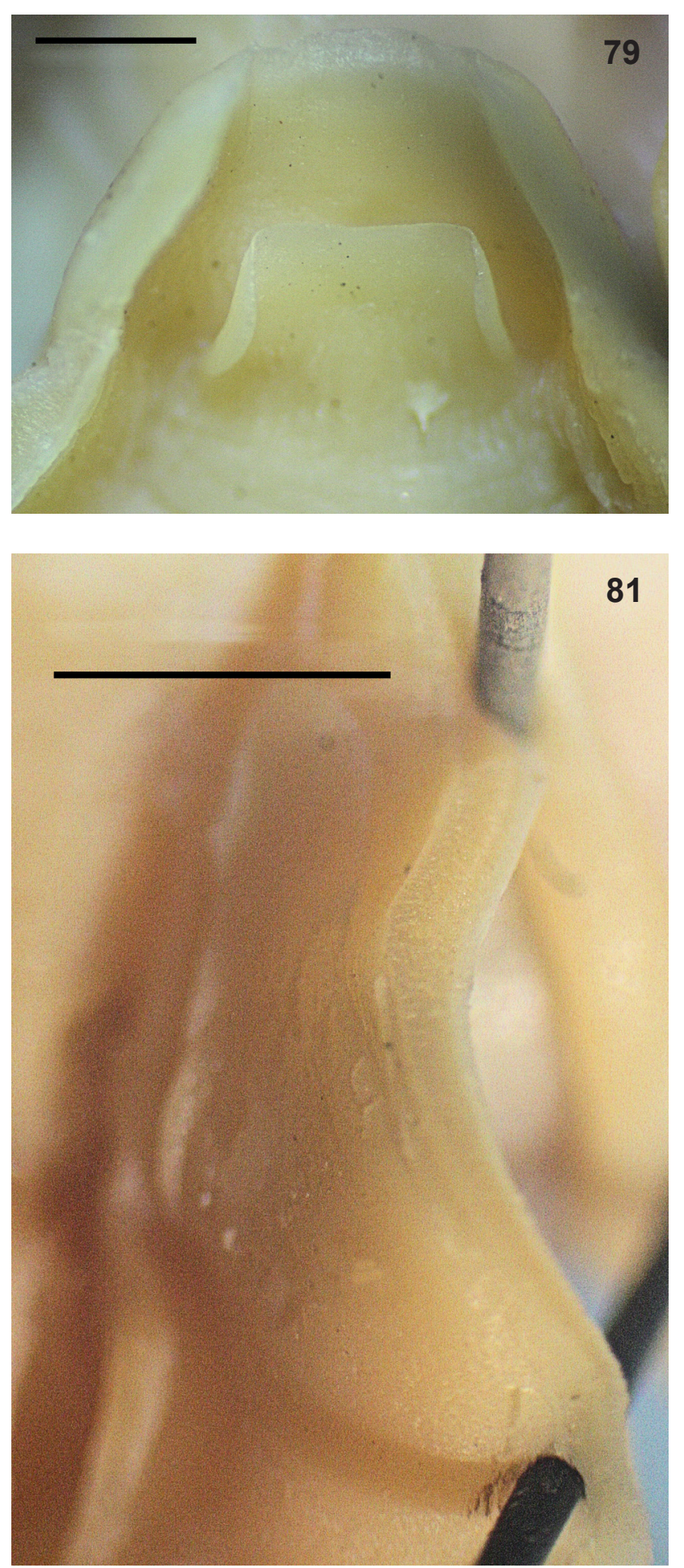

foundly from $S$. faurei in their size at maturity, their cuttlebones, and in the quadri-serial arrangements of suckers on arms (ROELEVELD 1972).

ROELEVELD (1972: table 6) compared S. robsoni, S. dubia and S. faurei. Her account of S. dubia "dorsal papillae" was subsequently re-evaluated and

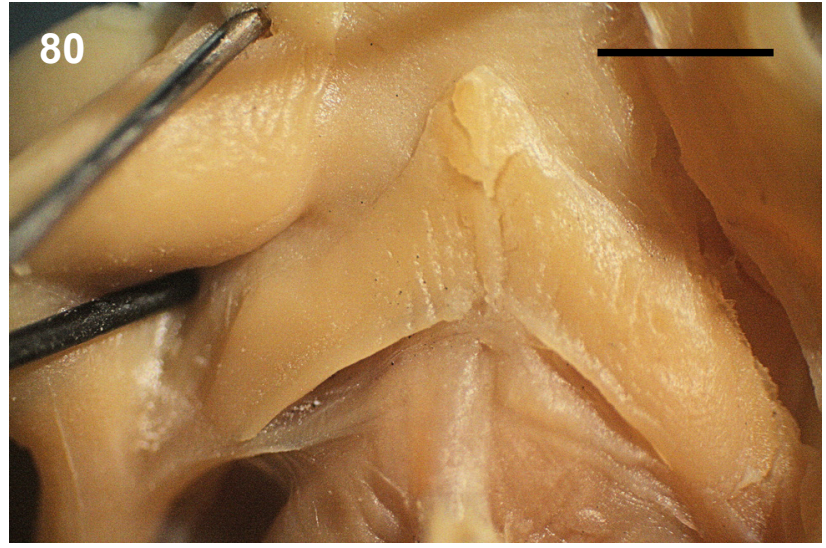

Figs 79-81. Sepia faurei, funnel organ, SAIAB 209589, female 21 mm ML: 79 - funnel valve; 80 - dorsal component; 81 - ventral component. Scale bars $1 \mathrm{~mm}$ (79), 2 $\mathrm{mm}(80-81)$

illustrated by LIPIŃSKI \& LESLIE (2018) who redescribed S. dubia. Concerning the interbrachial web of S. faurei, it was correctly described in ROELEVELD's table (1972: table 6), but not in the text of her description. It is now illustrated (Fig. 59). The tips of arms I are illustrated to show differences between $S$. robsoni and $S$. faurei based on the greater number of specimens (Figs 25-27, 61-63; compare ROELEVELD 1972: table 6). RoELEVELD (1972: fig. 16a) well illustrated the most common structure of the tip of I pair of arms of $S$. faurei, but there is, as in other species of the subgenus Digitosepia, considerable intra-specific variation. Tentacular clubs are well compared by ROELEVELD (1972: table 6) among the three species, but not the cuttlebone. In particular, the $S$. faurei cuttlebone is not "completely chitinous" - on the contrary, it is strongly calcified (Figs 82-83). The cuttlebone of the holotype must have been completely de-calcified, possibly because it was stored in formalin from capture (1902) to the description (early 70s').

I have provided photographs of the holotype of Sepia (D.) faurei for comparisons and future reference (Appendix, Fig. A2).

Distribution. Currently known from the small area of eastern Agulhas Bank (Fig. 1), with the depth range of $116-184 \mathrm{~m}$. Therefore, it may be a species of the continental shelf. It is interesting to note that the ranges of $S$. faurei, S. barosei, S. roeleveldi and $S$. robsoni strongly overlap in one small area off the Tsitsikamma coast. Three of these uncommon species were taken by a single trawl! 


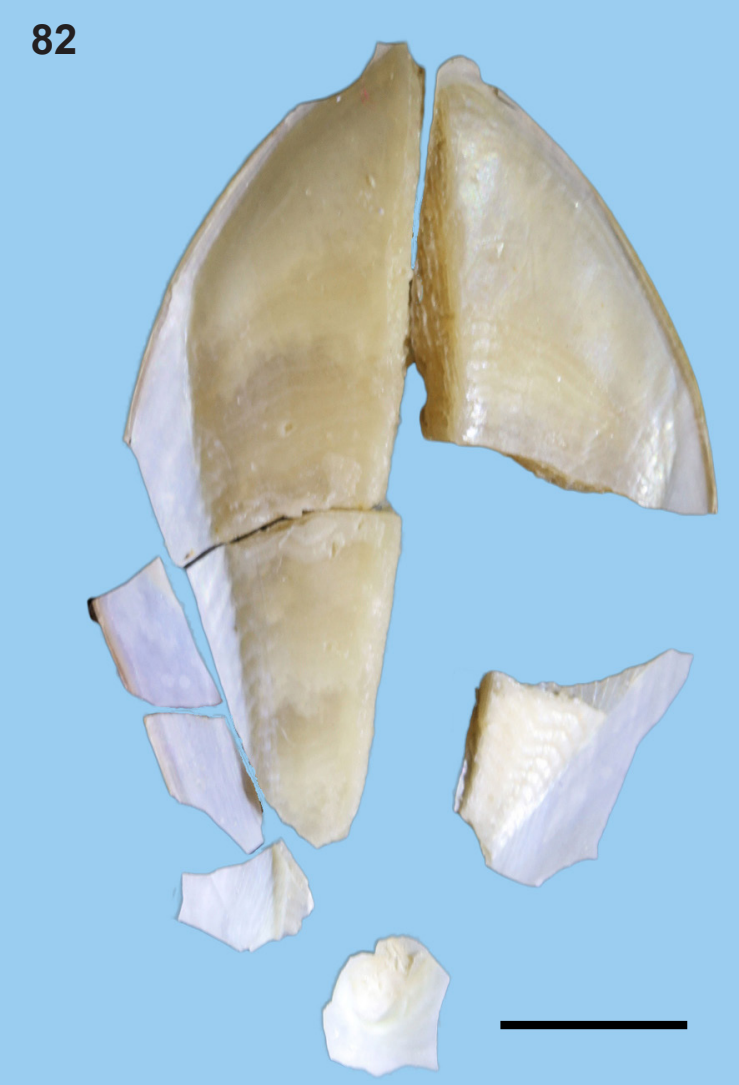

\section{3}
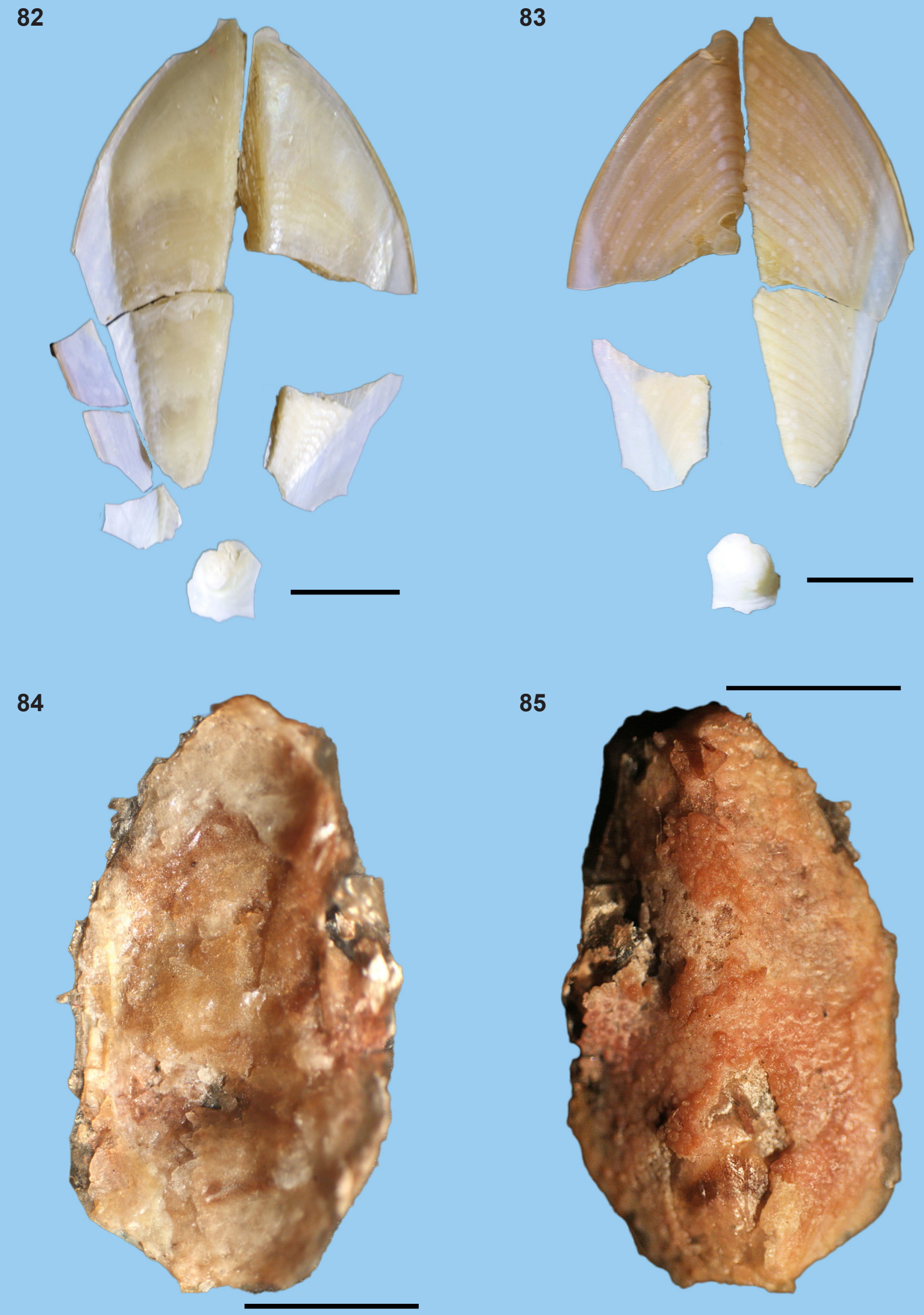

85

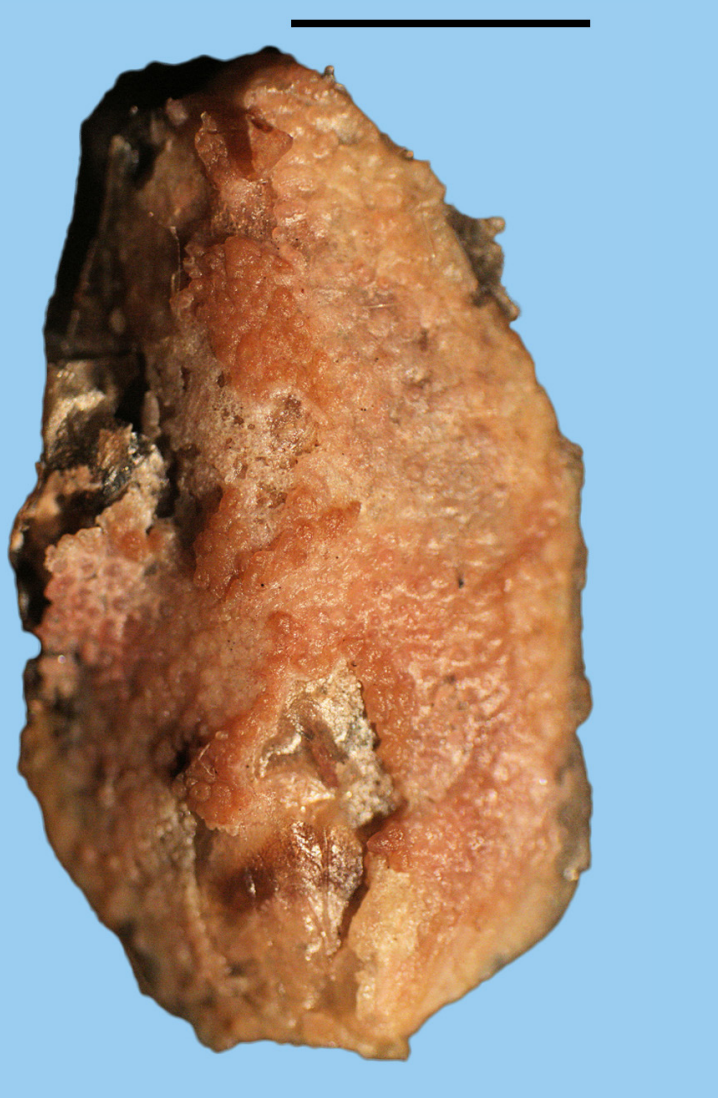

Figs 82-85. Sepia faurei, cuttlebone: 82-83 - SAMC MB A089325, female 29 mm ML: 82 - ventral view, 83 - dorsal view; 84-85 - SAIAB 209588, female 20 mm ML: 84 - ventral view, 85 - dorsal view. Scale bars 5 mm 


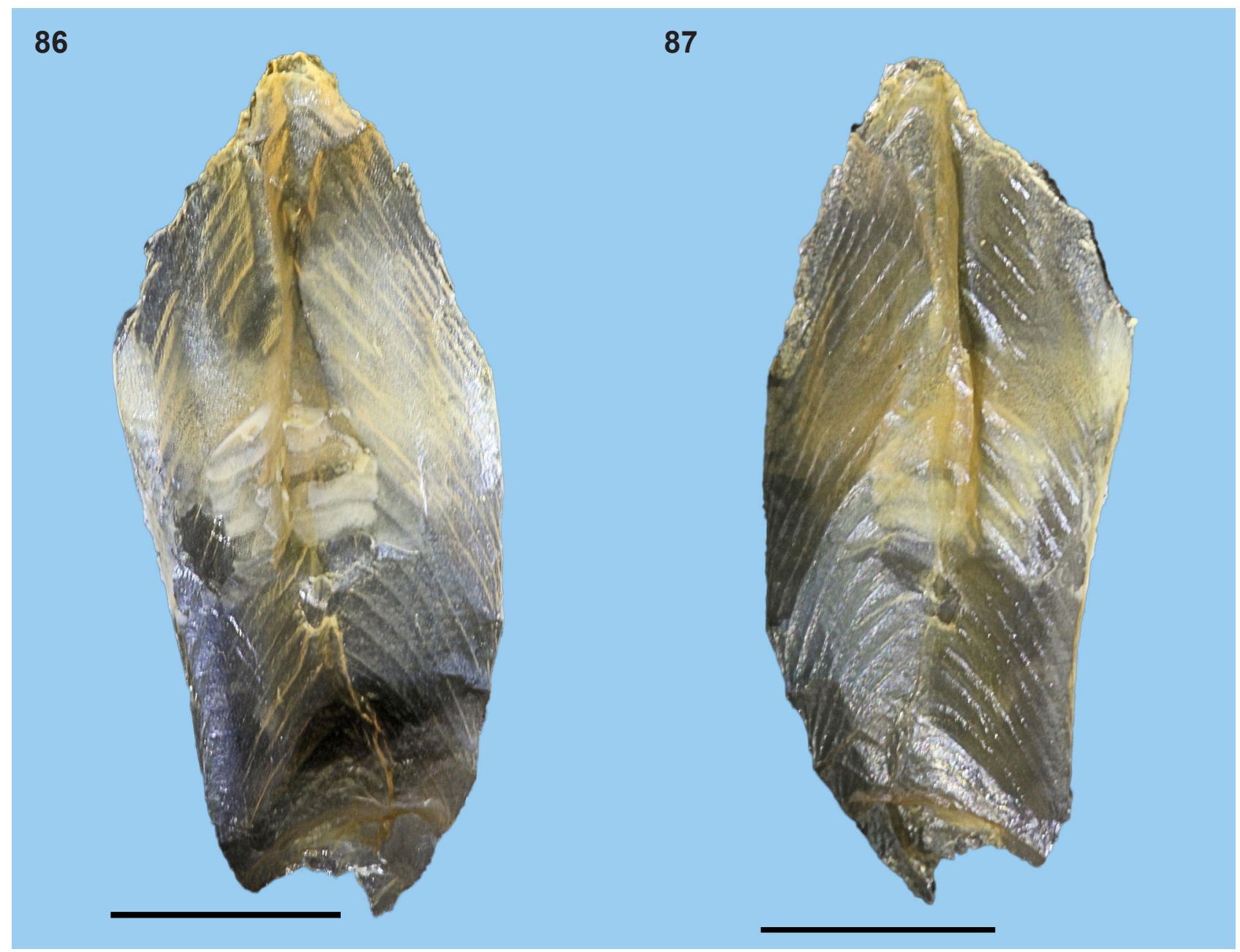

Figs 86-87. Sepia faurei, cuttlebone: 86-87 - SAIAB 209589, female 21 mm ML: 86 - ventral view, 87 - dorsal view. Scale bars $5 \mathrm{~mm}$

\section{DISCUSSION}

With this detailed account of the morphology and systematic status of S. robsoni and S. faurei, the definition of the recently described subgenus Digitosepia (LIPIŃSKI 2020) is better founded and the specific characters illustrated. Particularly important was the preparation of a fairly intact and only slightly damaged cuttlebone of S. robsoni (Figs 42-43), the individual of which, after capture, was preserved in $70 \%$ ethyl alcohol only. The same applies to the specimens of $S$. faurei SAMC (stored only in ethyl alcohol; however, since these voucher specimens were intended for molecular analysis and genetic interpretation, the alcohol was $96 \%$ which made them hard and therefore difficult to handle and describe). The accumulated collection of cuttlebones of four species of Digitosepia, despite its serious shortcomings (on the whole, poor preservation of the material) allows their characteristics to be summarised as follows:

Each species of Digitosepia has a different degree of calcification (understood simply as a build-up of cal- cium carbonate in a structure) - from extremely light (as in S. robsoni) to thick and heavy (as in S. faurei). This is a species-specific, environment-driven adaptation to extremely diverse environments in which these species live (DENTON \& GILPIN-BROWN 1961). This interpretation, as well as the molecular analysis, led BONNAUD et al. (2006: 148-149) to conclude that "the shell [cuttlebone - MRL] shape classically used as diagnostic of a lineage is not phylogenetically informative because of its phenotypic plasticity." Instead, they argued, that the "ventral part of the inner cone, seems robust in establishing the relationships of species in this group" [Sepia-MRL].

The postero-ventral (facing downwards when the animal swims) part of the inner cone has, on the other hand, fairly uniform characters in Digitosepia: it is fully integrated, narrow, flat (i.e. not protruding above the surface) and fused with the surrounding parts of the cuttlebone (LIPIŃSKI 2020: figs 33-34, 69-71; this contribution: Figs 42-47, 82-87). The 
forked limbs are thin, reduced, and fully integrated (as in S. (D.) barosei and S. (D.) roeleveldi) or absent (as in S. (D.) robsoni and S. (D.) faurei).

Septa of the phragmocone and striae of the dorsal shield are convex (straight in the middle part in $S$. (D.) robsoni), and not wavy in any part of the structure (as in S. (S.) typica).

The term "calcification" or "[degree] of calcification" is frequently used in descriptions of cuttlebones. However, various authors may use this term not exactly meaning the chemical process and its result, as most commonly defined accumulation of calcium salts in tissues, or deposition involving $\mathrm{CaCO}_{3}$ in biological systems (see The Free Dictionary by Farlex [FREE DICTIONARY 2003] under "calcification" and WEINER \& ADDADI 2011). For example, when describing Sepia pulchra Roeleveld et Liltved, 1985, ROELEVELD \& LILTVED (1985: 12) stated that "The shell is not calcified and is broadly oval...", whereas their figs 10-11 show the cuttlebone relatively hard and rigid, full of calcium carbonate (I have examined the cuttlebone of the holotype and can confirm this). In this context, "not calcified" means that a proper, thick, structured phragmocone is not present. Although classifying various degrees of "calcification" of cuttlebones is outside the scope of this paper, there is certainly a need for such a study, following the structural and physiological background provided by DENTON \& GILPIN-BROWN (1961) and SHERRARD (2000), systematic background of LU (1998), evolutionary background of BONNAUD et al. (2006) and a general, physiological approach of WEINER \& ADDADI (2011). S. (D.) robsoni is particularly interesting and potentially of a great value for research of the cuttlebone calcification process in the sepiids. The last anterior septum of the phragmocone of this species shows a remarkable dot calcification on the surface of the dorsal shield (Fig. 47); whilst in other sepiids this septum is most heavily calcified (LIPIŃSKI 2020: figs 36-37); in S. (D.) robsoni it is the least calcified.

\section{REFERENCES}

AXELSEN B. E., JOHNSEN E. 2014. An evaluation of the bottom trawl surveys in the Benguela Current Large Marine Ecosystem. Fisheries Oceanography 24 (Suppl. 1): 74-87. https://doi.org/10.1111/fog.12079

BONNAUD L., LU C. C., BOUCHER-RODONI R. 2006. Morphological character evolution and molecular trees in sepiids (Mollusca: Cephalopoda): is the cuttlebone a robust phylogenetic marker? Biological Journal of the Linnean Society 89: 139-150. https://doi.org/10.1111/ j.1095-8312.2006.00664.x

DENTON E. J., GILPIN-BRown J. B. 1961. The buoyancy of the cuttlefish Sepia officinalis (L.). Journal of the Marine
LIPIŃSKI \& LESLIE (2018: 145) stated that “[small sepiids] are so different from one another that virtually the only trait that they share is their small size, and this trait alone cannot have any systematic significance." However, it has great systematic significance when combined with other characters. For example, individuals of S. joubini Massy, 1927 are also small but they differ from the whole Digitosepia group in the sucker arrangement on the arms. The small size at sexual maturity, bi-serial sucker arrangement on the arms, cuttlebone characters and possibly also modifications of IV arms in males, may be sufficient to grant a separate generic status to the small, near-endemic cuttlefishes of southern Africa. However, to confirm this, a full molecular evaluation will be necessary.

\section{ACKNOWLEDGEMENTS}

I am grateful to my sea-going colleagues (notably Ms SHAZ DU PLESSIS \& Mr ODDGEIR ALVHeIM) for taking part in the collection of the material for this study. I thank Dr Roger BILLS \& Mr NKOSINATHI MAZUngula (SAIAB) and Mr Albé Bosman (Iziko Museum) for their help with the accessioned material, Dr VlAD LAPTIKHOVSKY for drawing the map (Fig. 1), and Ms CATHY BOUCHER for helping with two other Figures. Dr DIRK FUCHS helped greatly with the cuttlebone interpretations. Dr JON ABLETT measured the holotype of Sepia robsoni and provided its photographs (the copyright rests with the NHM). Finally, my most sincere thanks go to our involved colleagues from the Institute of Marine Research (Bergen, Norway), FAO (Rome, Italy), and the former Department of Agriculture, Forestry and Fisheries (DAFF; Cape Town, South Africa), especially the late Mr TORE StrøMme and Dr Rob LESLIE, for funding and/or logistical support, and friendship. And I thank my wife Aniela for her patience.

Biological Association of the U.K. 41: 319-342. https:// doi.org/10.1017/S0025315400023948

FREE Dictionary 2003. The free dictionary by Farlex. Available at https://www.thefreedictionary.com/ (accessed 25 June 2020)

LIPIŃSKI M. R. 2020. Two new species of Sepia Linnaeus, 1758 (Cephalopoda: Sepiidae) from South African waters. Folia Malacologica 28: 175-200. https://doi. org/10.12657/folmal.028.013

LIPIŃSKI M. R., LESLIE R. W. 2018. A new species of Sepia (Cephalopoda: Sepiidae) from South African waters with a re-description of Sepia dubia Adam et Rees, 
1966. Folia Malacologica 26: 125-147. https://doi. org/10.12657/folmal.026.014

LipińsKi M. R., NAgGs F. A., Roeleveld M. A. C. 2000. Catalogue of types of recent cephalopods in the collection of the Natural History Museum, London. Annales Zoologici (Warszawa) 50: 101-120.

LIPIŃSKI M. R., UNDERHILL L. G. 1995. Sexual maturation in squid: quantum or continuum? South African Journal of Marine Science 15: 207-223. https://doi. org/10.2989/02577619509504844

MASSY A. L. 1927. The Cephalopoda of the South African Museum. Annals of the South African Museum 25: 151-167.

LU C. C. 1998. Use of the sepion in the taxonomy of Sepiidae (Cephalopoda: Sepioidea) with an emphasis on the Australian fauna. In: Voss N. A., VeCCHIONE M., TOll R. B., SweEnEY M. J. (eds). Systematics and biogeography of Cephalopods. Vol. I. Smithsonian Contributions to Zoology 586: 207-214.

LU C. C., REID A. 1997. Two new cuttlefishes (Cephalopods: Sepiidae) from the North West Shelf, and a redescrip- tion of Sepia sulcata Hoyle, 1885. Records of the Western Australian Museum 18: 277-310.

RoEleveld M. A. 1972. A review of the Sepiidae (Cephalopoda) of southern Africa. Annals of the South African Museum 59: 193-313, plates 35-45.

Roeleveld M. A., LiLTVED W. R. 1985. A new species of Sepia (Cephalopoda, Sepiidae) from South Africa. Annals of the South African Museum 96: 1-18.

SHERRARD K. M. 2000. Cuttlebone morphology limits habitat depth in eleven species of Sepia (Cephalopoda: Sepiidae). Biological Bulletin 198: 404-414. https:// doi.org/10.2307/1542696

WeINER S., ADDADI L. 2011. Crystallization pathways in biomineralization. Annual Review of Materials Research 41: 21-40. https://doi.org/10.1146/annurev-matsci-062910-095803

Received: July 4th/August 14th, 2020 Revised: October 10th, 2020 Accepted: October 25th, 2020 Published on-line: November 23rd, 2020 


\section{APPENDIX}

HOLOTYPES OF SEPIA ROBSONI AND S. FAUREI

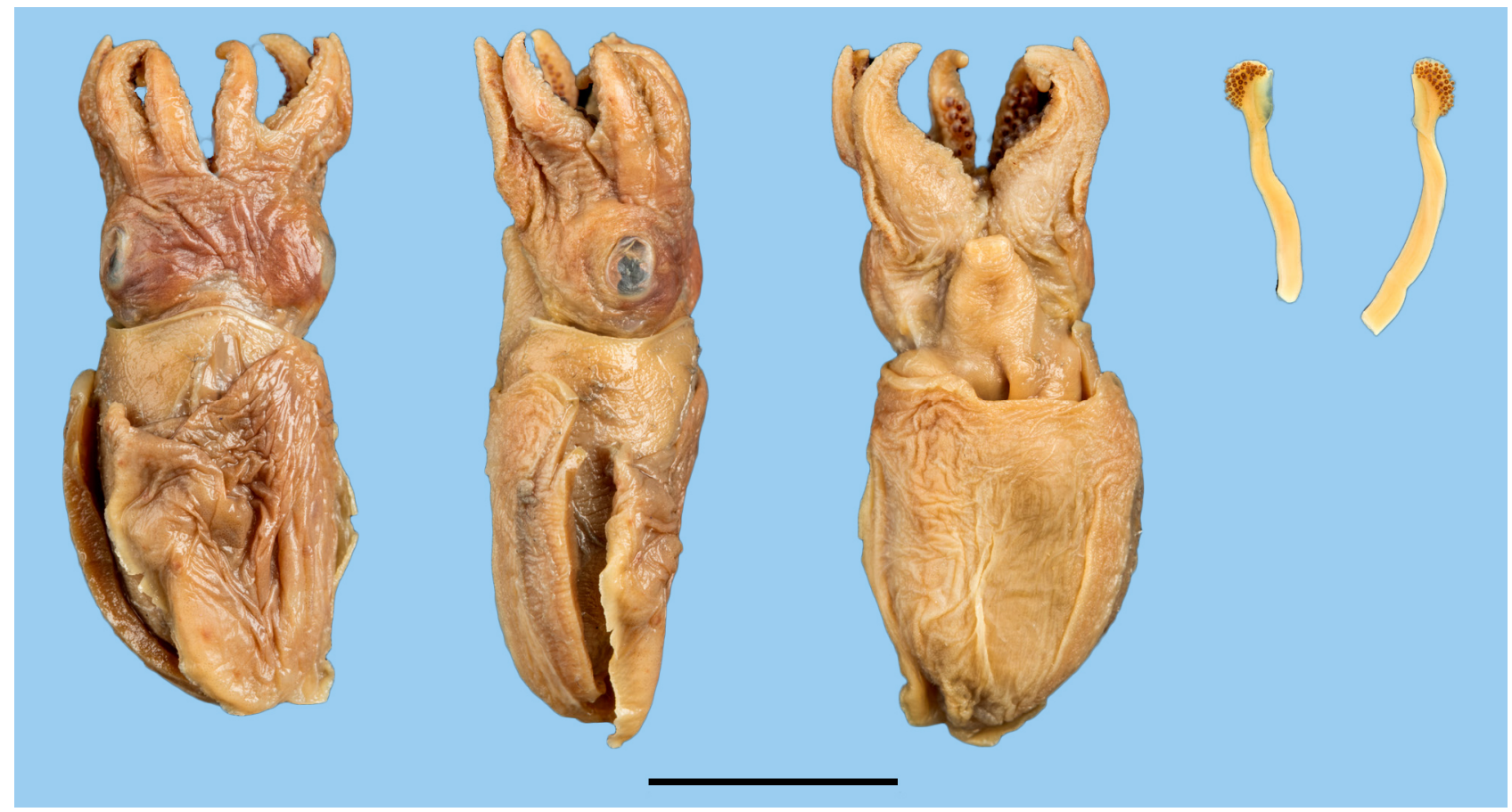

Fig. A1. Holotype of Sepia robsoni: dorsal, lateral, ventral view, and tentacles. Photo: NHM London. Scale bar $10 \mathrm{~mm}$

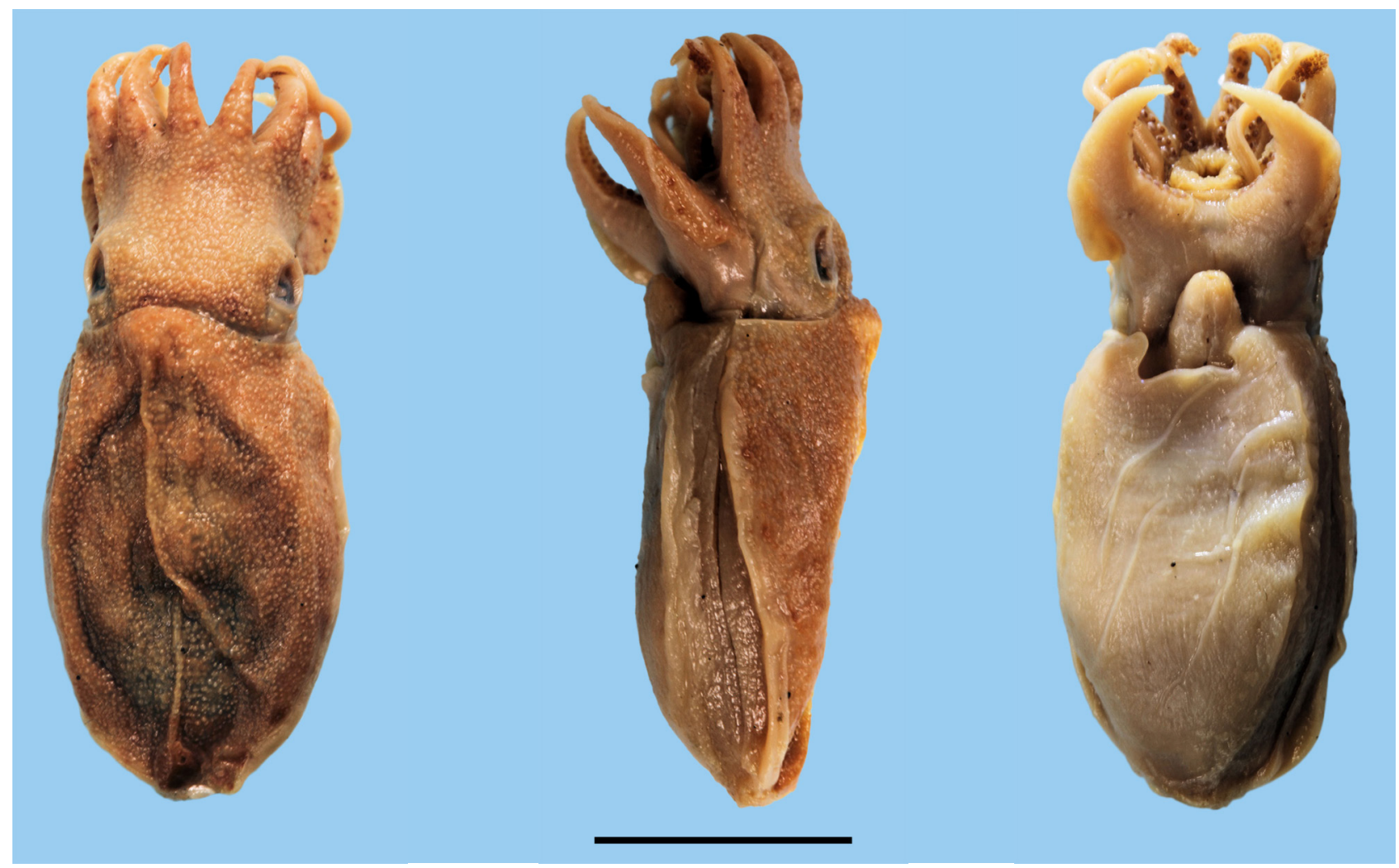

Fig. A2. Holotype of Sepia faurei: dorsal, lateral, ventral view. Scale bar $10 \mathrm{~mm}$ 